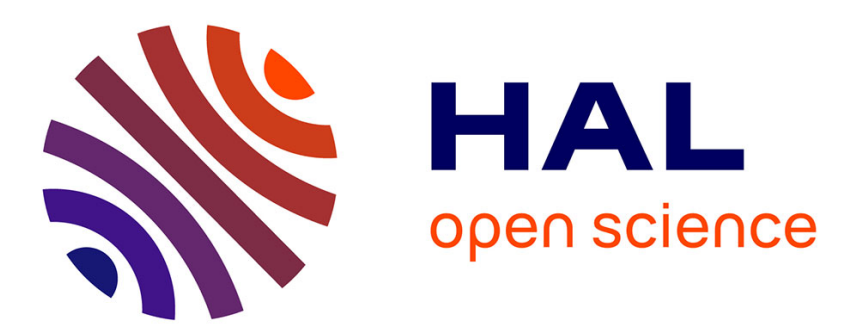

\title{
On the measurement of the bend elastic constant in nematic liquid crystals close to the nematic-to-SmA and the nematic-to-N TB phase transitions
}

P. Oswald, J. Colombier

\section{- To cite this version: \\ P. Oswald, J. Colombier. On the measurement of the bend elastic constant in nematic liquid crystals close to the nematic-to-SmA and the nematic-to-N TB phase transitions. Liquid Crystals, 2021, pp.1-25. 10.1080/02678292.2021.1896041 . hal-03215320}

\author{
HAL Id: hal-03215320 \\ https://hal.science/hal-03215320
}

Submitted on 3 May 2021

HAL is a multi-disciplinary open access archive for the deposit and dissemination of scientific research documents, whether they are published or not. The documents may come from teaching and research institutions in France or abroad, or from public or private research centers.
L'archive ouverte pluridisciplinaire HAL, est destinée au dépôt et à la diffusion de documents scientifiques de niveau recherche, publiés ou non, émanant des établissements d'enseignement et de recherche français ou étrangers, des laboratoires publics ou privés. 


\title{
Original Article
}

\section{On the measurement of the bend elastic constant in nematic liquid crystals close to the nematic-to-SmA and the nematic-to- $N_{T B}$ phase transitions}

\author{
P. Oswald*, J. Colombier \\ Univ Lyon, Ens de Lyon, Univ Claude Bernard, CNRS, Laboratoire de Physique, F-69342 Lyon, France.
}

(v1.0 released December 2020)

\begin{abstract}
We propose to measure the ratio $\kappa_{31}$ of the bend elastic constant $K_{3}$ over the splay elastic constant $K_{1}$ of a nematic phase by using an optical method based on the measurement of the birefringence of homeotropic/planar hybrid samples. This method is compared to the classical measurements using the Freedericksz transition under AC electric field and its advantages and disadvantages are discussed. Both methods are applied to the measurement of $K_{1}$ and $K_{3}$ close to the nematic-to-SmA phase transition of the liquid crystal $8 \mathrm{CB}$ and the nematic-to- $\mathrm{N}_{\mathrm{TB}}$ phase transition of the mixture $8 \mathrm{CB}+50 \mathrm{wt} \% \mathrm{CB} 7 \mathrm{CB}$. The role of flexoelectricity in these measurements is analyzed, as well as the role of the pretilt angle and the anchoring energy of the molecules on the plates limiting the samples.
\end{abstract}

Keywords: nematic liquid crystal; twist-bend nematic; smectic A; elastic constants; Freedericksz transition; flexoelectricity; pretilt angle; anchoring energy

\section{Introduction}

A few month ago, I (PO) proposed to a third-year student (JC) to measure the bend elastic constant $K_{3}$ in the nematic phase $[1,2]$ of the liquid crystal (LC) $8 \mathrm{CB}$ close to the smectic A phase (SmA) by using the Freedericksz transition $[1,2]$ and to compare these results with similar measurements performed close to the twist-bend nematic phase $\left(\mathrm{N}_{\mathrm{TB}}\right)$ of a mixture of $8 \mathrm{CB}$ and the dimer molecule CB7CB (for a review about the properties of the $\mathrm{N}_{\mathrm{TB}}$ phase, see Ref. [3]). These measurements are interesting in itself because $K_{3}$ diverges and becomes very large close to the SmA phase [2, $4]$, whereas it is very small and has a non-monotone behavior close to the $\mathrm{N}_{\mathrm{TB}}$ phase $[5,6]$. In practice, the experiment consists of measuring the capacitance of a planar sample as a function of the applied voltage at different temperatures, and then to fit these curves with the adequate theoretical model. In doing this, we realized how difficult it was to obtain accurate values of $K_{3}$ with this technique. The difficulties were both experimental and theoretical. Experimental because reliable capacitance curves must be measured, which is not so easy to achieve very close to a phase transition. Indeed, this requires that the temperature, but also the chemical composition of the LC, are perfectly homogeneous over the whole surface of the sample. In addition, special care must be taken to be sure that equilibrium curves are measured. This requires to increase the voltage very slowly, in particular close to the smectic A phase because of the divergence of the rotational viscosity [4]. This precaution must also be taken with the mixture with $\mathrm{CB} 7 \mathrm{CB}$, in which the rotational viscosity is larger than in usual nematics because of the large size of the CB7CB molecules. For these reasons,

*Corresponding author. Email: patrick.oswald@ens-lyon.fr 

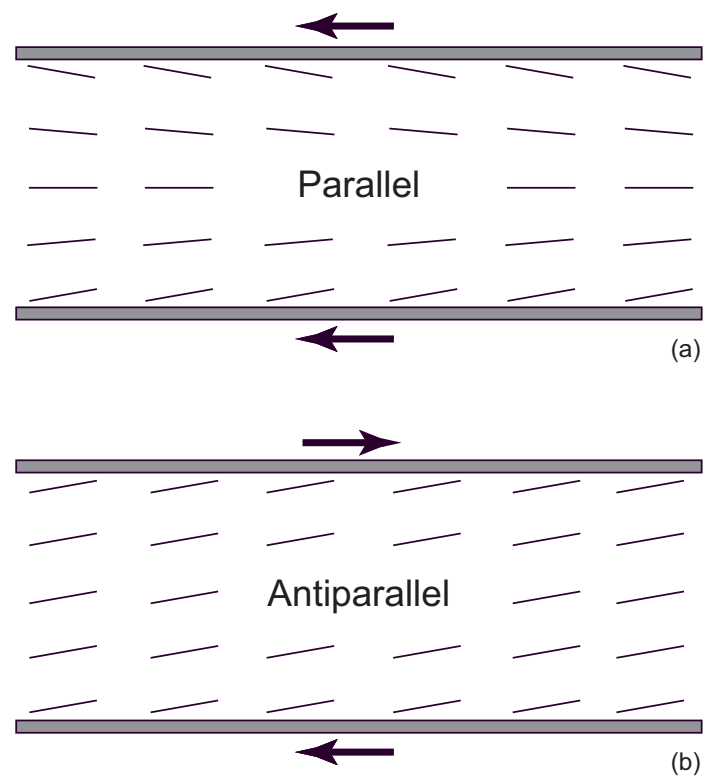

Figure 1. director filed in a 'parallel' (a) and 'antiparallel' (b) sample. The arrows indicate the rubbing direction of the polyimide layer on the plates.

one hour and sometimes more can be necessary to just measure a single capacitance curve, which makes the experiments very long. This can be a problem when the liquid crystal degrades or pollutes on contact with the glue used to stick the sample, or worse when one the compounds of the mixture crystallizes, which often produced with $\mathrm{CB} 7 \mathrm{CB}$ below the clearing point. The difficulties are also theoretical, in particular because of the great number of fit parameters. If the samples were perfectly planar and the anchoring strength infinite, these parameters would only be the critical voltage $V_{c}$ of the Freedericksz transition, the two dielectric constants $\epsilon_{\|}$and $\epsilon_{\perp}$ parallel and perpendicular to the director and the two splay and bend elastic constants $K_{1}$ and $K_{3}$. In this situation, $\epsilon_{\perp}$ is obtained from the capacitance measured below the onset of instability while $\epsilon_{\|}$is obtained by extrapolating the capacitance at infinite voltage. As for $K_{1}$, it is obtained by measuring the critical voltage $V_{c}$ while $K_{3}$ is obtained by analyzing the shape of the curve of capacitance above the onset of instability. In principle, this procedure is straightforward and should not pose any problems. In practice, this is not the case because the samples are not perfect. The first complication comes from the fact that the samples are not necessarily perfectly planar. This is the case in our experiments in which the planar anchoring was obtained by rubbing a thin layer of polyimide deposited on the electrodes. In that case, there is a small pretilt angle $\phi_{a}$ and the capacitance curves change depending on whether the two ITO electrodes are rubbed in the same or in opposite directions. In the following, we will call 'parallel samples' the samples in which the two electrodes are rubbed in the same direction and 'antiparallel samples' the samples in which the electrodes are rubbed in opposite directions 1. It turns out that there still exists a well-defined critical voltage $V_{c}^{\prime}$ in parallel samples, whereas the director field is always unstable under electric field in antiparallel samples whatever the anchoring strength. The situation becomes still more complicated if one takes into account the anchoring energy $W_{a}$, adding the extrapolation length $l_{a}=W_{a} / K_{1}$ [1] to the list of the fit parameters. Finally, it has been stressed out recently that flexoelectric effects could also play an important role by renormalizing the value of $K_{3}$ [7] as in the ferroelectric $\mathrm{SmC}^{\star}$ phase [8]. This effect could bias the measurements of $K_{3}$ when it becomes very small, as emphasized by the authors of Ref. [5]. This brief review shows that fitting a capacitance curve to obtain $K_{3}$ is not easy if we wish to take into account all the parameters, namely, the two dielectric constants, the critical voltage $V_{c}$ (or $K_{1}$ ), the elastic anisotropy coefficient $\kappa_{31}=K_{3} / K_{1}$, the pretilt angle $\phi_{a}$, the anchoring energy $W_{a}$ (or the extrapolation length $l_{a}=K_{1} / W_{a}$ ) and the bulk flexoelectric coefficient $e^{\star}=e_{1}+e_{3}$ (by using the conventions of Bob Meyer for the flexoelectric coefficients $e_{1}$ and $e_{3}[9]$ ). This is nonetheless not always necessary as we shall see later, on condition to use the right type of sample. Because of the complexity of the 


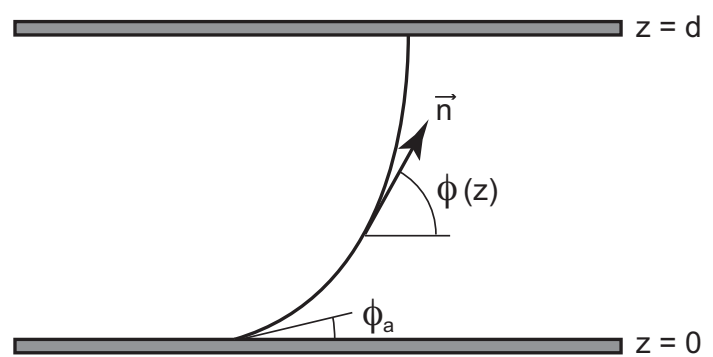

Figure 2. Hybrid sample geometry. The $z$-axis is perpendicular to the glass plates.

capacitance measurements, we looked for another method to measure the elastic anisotropy $\kappa_{31}$, which does not need to use an electric field. The fully optical method we propose in this paper is based on the measurement of the birefringence of homeotropic/planar hybrid samples. It resembles the interferometric method used by Scudieri to measure $\kappa_{31}$ in cylindrical capillaries [10, 11], but it is simpler to implement as it does not need to use a reference sample.

The goal of this paper is to present and compare this method with the standard method by capacitance measurements and to apply it to the measurement of the elastic anisotropy $\kappa_{31}$ in pure $8 \mathrm{CB}$ and in the mixture $8 \mathrm{CB}+50 \mathrm{wt} \% \mathrm{CB} 7 \mathrm{CB}$.

The paper will consist of three parts and three appendices. In the first part (Section 2) the principle of the optical method is described. In this part, the flexoelectric effects will be neglected and the anchoring strength will be supposed infinite on the two surfaces. In the second part (Section 3) the experimental setup is described and the optical measurements are detailed. Finally, our results are compared to those obtained by using the Freedericksz transition in the third part (Section 4). The main formulas about the Freedericksz transition in parallel and antiparallel samples are recalled in Appendix A. The role of flexoelectric effects is discussed in Appendix B. Finally, the role of the anchoring energy is discussed in Appendix $\mathrm{C}$ in which a new method to measure the anchoring energy is proposed.

\section{Principle of the optical method}

The optical method we used to measure $\kappa_{31}$ is very simple and consists of measuring the path difference between the ordinary and extraordinary rays propagating across a nematic cell treated for planar anchoring on one plate and for homeotropic anchoring on the opposite one. In such a hybrid sample (Fig. 2), the director field distorts in such a way to minimize the elastic energy

$$
F_{e}=\int_{0}^{d} \frac{1}{2} K(\phi)\left(\frac{\mathrm{d} \phi}{\mathrm{d} z}\right)^{2} \mathrm{~d} z
$$

In this equation, $\phi$ denotes the angle between the director and the plates, the $z$-axis is perpendicular to the glass plates and $d$ is the sample thickness, and $K(\phi)=K_{1} \cos ^{2} \phi+K_{3} \sin ^{2} \phi$. Note that flexoelectric effects are neglected here for simplicity. This approximation will be discussed in Appendix B. Minimization of $F_{e}$ with respect to $\phi$ gives the torque equation

$$
\left(1+\kappa \sin ^{2} \phi\right) \frac{\mathrm{d}^{2} \phi}{\mathrm{d} z^{2}}+\kappa \sin \phi \cos \phi\left(\frac{\mathrm{d} \phi}{\mathrm{d} z}\right)^{2}=0
$$

where $\kappa=\kappa_{31}-1$. Solving this equation with the boundary conditions

$$
\begin{cases}\phi(0)=\phi_{a} & \text { (planar anchoring) } \\ \phi(d)=\frac{\pi}{2} & \text { (homeotropic anchoring) }\end{cases}
$$




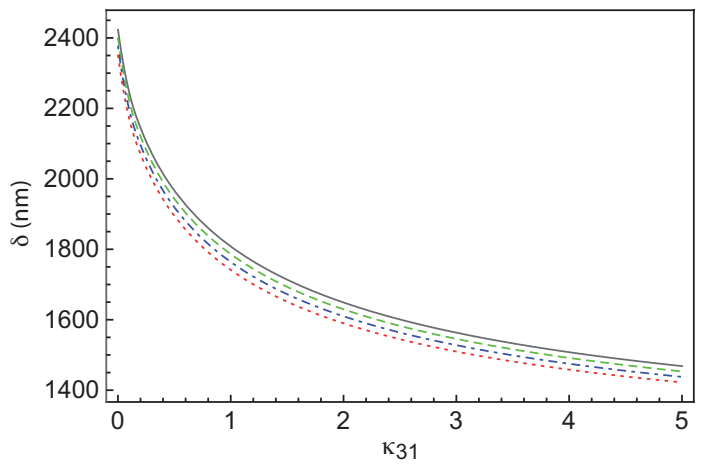

Figure 3. Retardation $\delta$ as a function of $\kappa_{31}$ calculated for different values of $\phi_{a}$ by taking $n_{o}=1.5, n_{e}=1.65$ and $d=25 \mu \mathrm{m}$. From top to bottom, $\phi_{a}=0,1,2,3^{\circ}$.

gives the director profile $\phi(z)$ from which the path difference between the ordinary and extraordinary rays can be calculated by using the formula

$$
\delta=\int_{0}^{d}\left[\frac{1}{\sqrt{\frac{\sin ^{2} \phi}{n_{o}^{2}}+\frac{\cos ^{2} \phi}{n_{e}^{2}}}}-n_{o}\right] \mathrm{d} z
$$

In these formulas, $n_{o}$ is the ordinary index, $n_{e}$ is the extraordinary index and $\phi_{a}$ is the pretilt angle. Note that we assumed strong anchoring conditions on the two plates, a point that is discussed in Appendix C.

In practice, the torque equation (2) subjected to the boundary conditions (3) can be easily solved using Mathematica. From this solution, the path difference $\delta$ can be calculated by using Eq. (4). In Fig. 3, we plotted $\delta$ as a function of $\kappa_{31}$ by taking $\lambda=632.8 \mathrm{~nm}$ (He-Ne laser light) with $n_{o}=1.5$, $n_{e}=1.65$ and $d=25 \mu \mathrm{m}$ which are typical values in our experiments and $\phi_{a}=0,1,2,3^{\circ}$. This example shows that $\delta$ is very sensitive to the value of $\kappa_{31}$ but also depends on $\phi_{a}$. As a consequence, measuring $\delta$ should allow us to measure $\kappa_{31}$ providing $\phi_{a}$ is known. This is the principle of the optical method.

\section{Optical measurements of $\kappa_{31}$}

\subsection{Sample preparation and experimental setup}

In practice, the samples were prepared between two ITO glass plates. The top plate was treated for homeotropic anchoring with the polyimide Nissan SE-4811 and the bottom one was treated for planar anchoring with the polyimide Nissan 0825. Both polyimides (PI) were deposited by spincoating and then baked at high temperature $\left(180^{\circ} \mathrm{C}\right.$ for the PI 0825 and $280^{\circ} \mathrm{C}$ for the PI SE-4811) for 30 to $45 \mathrm{~min}$. For the plates treated for planar anchoring, the anchoring direction was obtained by rubbing their surface in a single direction with a soft velvet fabric. For this purpose, a machine specially designed by Artyom Pretrossian was used. It allowed us to prepare all the plates in the same experimental conditions. This was important to always obtain the same pretilt as we know that the latter depends on the rubbing conditions [12]. Nickel wires were used as a spacer to fix the sample thickness and an epoxy glue was used to stick them. A special care was taken to the parallelism between the two glass plates, better than $510^{-5} \mathrm{rad}$ in all the samples. The samples were placed in a home-made oven regulated to within $\pm 2 \mathrm{mK}$ thanks to a PID controller ATNE ATSR 100. The sample temperature was measured with a Pt100 sensor placed in the chamber containing the sample. The sample was placed in a frame made in fiberglass that could be moved in the horizontal plane from outside thanks to two micrometer translation stages. Because of this frame, there exists a small temperature gradient inside the chamber containing the sample of the order of 
$10 \mathrm{mK} / \mathrm{cm}$ at $40^{\circ} \mathrm{C}$. This oven is mounted on the circular rotatable stage of a Leitz Laborlux 12 Pol microscope equipped with a Berek rotating compensator 0989M. The latter was used to measure the path difference $\delta$ between the extraordinary and ordinary rays propagating across the nematic slab. All measurements were done in red light $(\lambda=632.8 \mathrm{~nm})$ by closing the aperture diaphragm of the condenser to the maximum to get parallel light. In practice, the measurements were performed between crossed polarizers at $45^{\circ}$ with respect to the slow axis of the nematic samples, which is parallel to the rubbing direction of the plates. In this configuration, the slow axis of the birefringent plate of the compensator is perpendicular by construction to the slow axis of the sample so that the retardation of the compensator subtracts to the retardation of the sample. In practice, the retardation $\delta$ is obtained (to within a multiple of $\lambda$ easy to determine when the sample thickness and the birefringence are known) by measuring the tilt angles $i_{1}$ and $i_{2}$ in both directions of the plate of the compensator that are necessary to get a maximum of extinction of the image observed in the microscope. From the sum $i_{1}+i_{2}$, the retardation is obtained thanks to a calibration table sold with the compensator. In our experiment the maximum of extinction was obtained by measuring the light intensity through a 50- $\mu$ m-in-diameter central zone of the image with a video camera Pike F 145B interfaced with a LabView program. In this way, and by repeating the measurement five times for each temperature, the sum $i_{1}+i_{2}$ can be obtained with an accuracy better than $0.5^{\circ}$ which gives the retardation $\delta$ to within $\pm 5 \mathrm{~nm}$. We note from now on that these measurements are rapid and local which is a great advantage with respect to global capacitive measurements which are very long and very sensitive to the temperature or chemical composition gradients, in particular close to phase transitions. The liquid crystals used were 8CB (4-octyl-4'-cyanobiphenyl) and the dimer molecule CB7CB (1,7-bis(4-cyanobiphenyl-4'-yl) heptane). Both were purchased at Synthon and used without further purification.

\section{$3.2 \quad$ Experimental procedure}

In practice, measuring $\delta$ was not sufficient to get $\kappa_{31}$. Indeed, we also needed to know the two indices $n_{o}$ and $n_{e}$ and the sample thickness with a very good accuracy to obtain a reliable value of $\kappa_{31}$. And the pretilt angle $\phi_{a}$ must also be known precisely even if it is small -of the order of $2^{\circ}$-according to Nissan specifications.

In order to measure the sample thickness and the index $n_{o}$, we used an Ocean Optics USB2000 spectrometer. We first measured the spectrum of the empty cell. Note that this measurement was done at the same place as the optical measurements to eliminate errors due to the lack of parallelism between the two glass plates. From this spectrum the cell thickness is usually calculated by using the formula

$$
d=\frac{\lambda_{m} \lambda_{n}(n-m)}{2\left(\lambda_{m}-\lambda_{n}\right)}
$$

where $\lambda_{m}$ and $\lambda_{n}$ mark the positions of two minima of the spectrum separated by $n-m$ fringes. From this formula, the thickness is obtained at best to within $\pm 0.1 \mu \mathrm{m}$, which is not precise enough. To improve our accuracy, we fitted the whole spectrum with a program written with Mathematica 12. In this program all the extrema of the spectrum are considered. By adjusting their positions with the positions of the extrema of the theoretical spectrum, a much better accuracy was obtained, of the order of $\pm 0.02 \mu \mathrm{m}$. With this precision, it was possible to detect tiny sample thickness variations as a function of temperature. For this measurement the sample was placed in a Mettler FP82 oven. We observed that the sample thickness slightly increased when the temperature increased, as one could expect from the coefficient of thermal expansion of the epoxy glue used to stick the wires (of the order of $100 \mu \mathrm{m} / \mathrm{m}^{\circ} \mathrm{C}$ ).

In order to measure the ordinary index of the LC, we prepared a homeotropic sample and we measured its thickness before and after filling with the LC. The first measurement gives $d$ and the second $n_{o} d$. To increase the contrast of the fringes, glass plates covered with an ITO layer were 
systematically used. Measurements were done as a function of temperature by placing the sample in a Mettler oven. In this way, a value of $n_{o}(T)$ averaged over the visible spectrum was obtained with an accuracy of \pm 0.01 . This accuracy is quite enough for our measurements because $\delta$ is very little sensitive to the exact value of $n_{o}$ as one can check numerically from Eq. (4).

We then measured the birefringence $\Delta n=n_{e}-n_{o}$ of the two LCs in red-light, at $\lambda=632.8 \mathrm{~nm}$. For this measurement, parallel planar samples were used. First the thickness of each sample was measured before filling with the LC. Each sample was then filled by capillarity in the isotropic phase with the LC and then placed in the precision oven. The retardation $\delta$ between the ordinary and extraordinary rays was then measured with the Berek compensator. From this measurement the birefringence was deduced by using formula (4) and by assuming that $\phi(z)=\phi_{a}(1-2 z / d)$, which turns out to be exact when $\phi_{a}<<1$, whatever the value of the elastic anisotropy $K_{3} / K_{1}$. Because the measured birefringence was very little dependent on the value of $\phi_{a}$, we took $\phi_{a}=2^{\circ}$ in our calculations, as given by Nissan in its catalog. In this way, we obtained $\Delta n$ with a relative accuracy of about $510^{-4}$.

Once the birefringence was known, we looked for a simple method to measure the pretilt angle and check a posteriori the value given by Nissan. Several methods have been proposed in the literature. One can cite, for instance, the rotating crystal method [13, 14], the magnetic null method [15], the reflectometric methods $[16,17]$ and more sophisticated methods as the one using heterodyne interferometry [18]. Unfortunately, we were not equipped to perform such optical measurements. Another method, which we used (see the next section), consisted of analyzing the capacitance curves vs. the applied voltage of antiparallel planar samples [19,20], but this method is cumbersome. For this reason, we looked for another method, simpler or more rapid to implement, which does not need to have a LCR bridge. This method consisted of making a planar sample in which the two glass plates were rubbed in the same direction on one half of the surface and in opposite directions on the other half. By measuring the path shift $\delta$ on each side of the line separating the two regions, the pretilt angle was found by using formula (4). In the calculations, we took $\phi(z)=\phi_{a}$ in the 'antiparallel' region and $\phi(z)=\phi_{a}(1-2 z / d)$ in the 'parallel' region. In practice, we measured $\delta$ in two regions close to each other in order that the sample thickness did not change significantly between these two regions. In our experiments, the angle between the two plates was less than $210^{-5}$ rad and the two regions were situated less than $\sim 200 \mu \mathrm{m}$ apart. This ensured a thickness variation of less than 4 $\mathrm{nm}$, whence an error on $\delta$ of less than $1 \mathrm{~nm}$, which is negligible.

Finally, we prepared hybrid samples. For each of them, we first measured their thickness. The samples were then filled with the LC by capillarity in the isotropic phase and then placed in the precision oven. The path difference $\delta$ was then measured as a function of temperature with the compensator. Finally, the anisotropic ratio $\kappa_{31}$ was calculated by solving numerically with Mathematica 12 the torque equation (2) and by looking for which value of $\kappa_{31}$ the value of $\delta$ calculated from Eq. (4) is equal to the experimental value. We emphasize that the calculations are rapid and much simpler than in the capacitive method described in the next section.

We end this paragraph by mentioning that all of our optical experiments were conducted with samples of typical thickness $25 \mu \mathrm{m}$.

\section{Experimental results}

Fig. 4 shows the variations of $n_{o}$ as a function of temperature in the two LCs studied. In the graph for $8 \mathrm{CB}$, the temperature is referred to $T_{N A}$-the transition temperature to the smectic phase- while for the mixture $8 \mathrm{CB}+50 \mathrm{wt} \% \mathrm{CB} 7 \mathrm{CB}$ the temperature is referred to $T_{N B}$-the transition temperature to the twist-bend nematic phase. In the two graphs, the transition temperature to the isotropic liquid is marked with a vertical dashed line. All these temperatures were measured by observing the samples in the microscope between crossed polarizers. In the planar samples, the transition temperature to the SmA phase $T_{N A}$ was taken as the temperature below which the nematic fluctuations disappeared in the microscope. This temperature was not always easy to determine by direct observation and for 

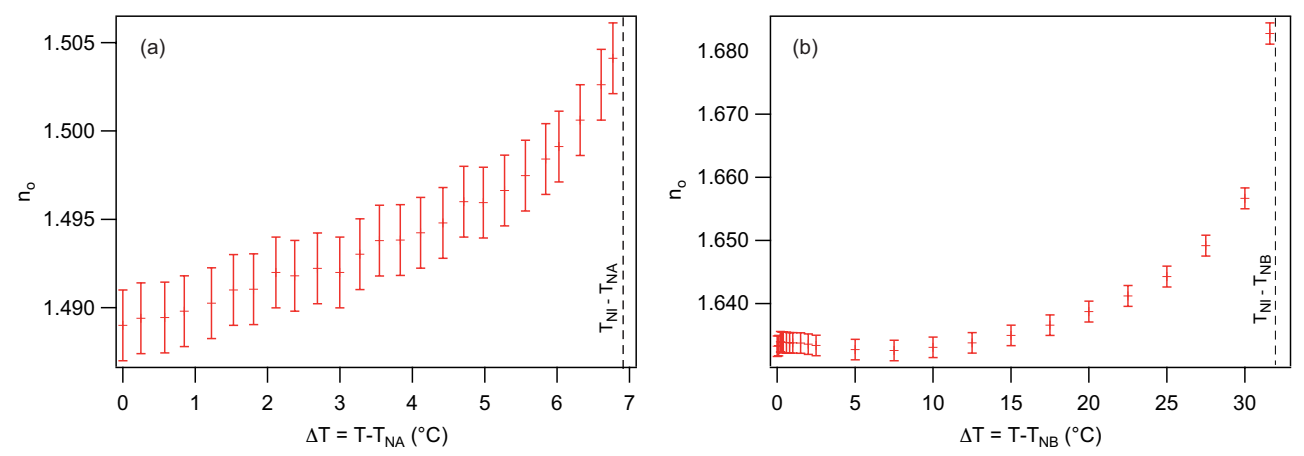

Figure 4. Ordinary index $n_{o}$ measured in pure $8 \mathrm{CB}$ and the mixture $8 \mathrm{CB}+50 \mathrm{wt} \% \mathrm{CB} 7 \mathrm{CB}$ as a function of temperature.
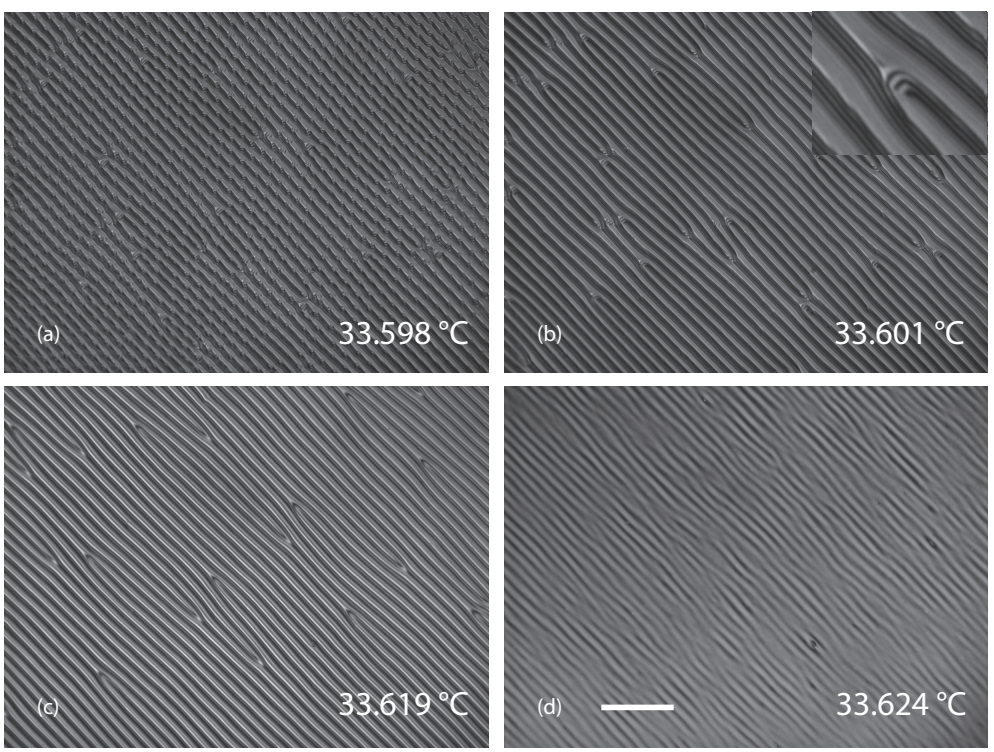

Figure 5. Temperature evolution of the banded texture observed in a hybrid sample of $8 \mathrm{CB}$ of thickness $d=21.34 \mu \mathrm{m}$. Focal conic domains are clearly visible at $33.598^{\circ} \mathrm{C}$. Between 33.600 and $33.930^{\circ} \mathrm{C}$ a banded texture forms in the sample with the band parallel to the rubbing direction. We have chosen for $T_{N A}$ the temperature at which a transverse modulation forms in the core of the dislocations of the banded texture as shown in the inset of the photo taken at $33.601^{\circ} \mathrm{C}$. Photos taken in red-light $(633 \mathrm{~nm})$ between crossed polarizers at $45^{\circ}$ of the bands. The white bar is $200 \mu \mathrm{m}$ long.

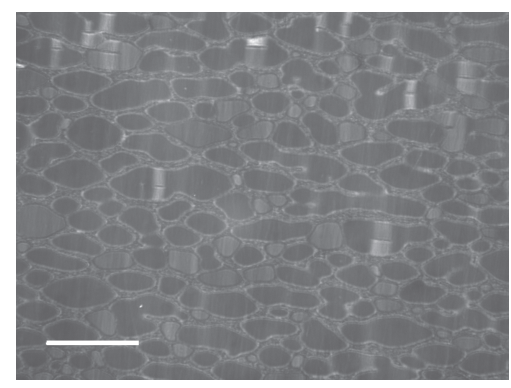

Figure 6. Domains of $\mathrm{N}_{\mathrm{TB}}$ phase coexisting with the nematic phase in a sample of the mixture $8 \mathrm{CB} / \mathrm{CB} 7 \mathrm{CB}$ of thickness $d=20 \mu \mathrm{m}$. Photo taken between crossed polarizers. The white bar is $100 \mu \mathrm{m}$ long.

this reason we used a trick consisting of subtracting two video images taken with a time interval of $\sim 1 \mathrm{~s}[21]$. In the nematic phase, the resulting image is very noisy because of the fluctuations of the director field. By contrast, the image is much less noisy in the SmA phase in which the fluctuations are much less important. In this way $T_{N A}$ was determined with an accuracy of $\pm 2.5 \mathrm{mK}$ in the planar samples. The situation was more complicated in the hybrid homeotropic/planar samples because of the presence of a band instability of the nematic texture when $T_{N A}$ is approached. This instability is due to the divergence of $K_{3}$ and was known for a long time [4, 22]. This texture, shown in Fig. 5, 

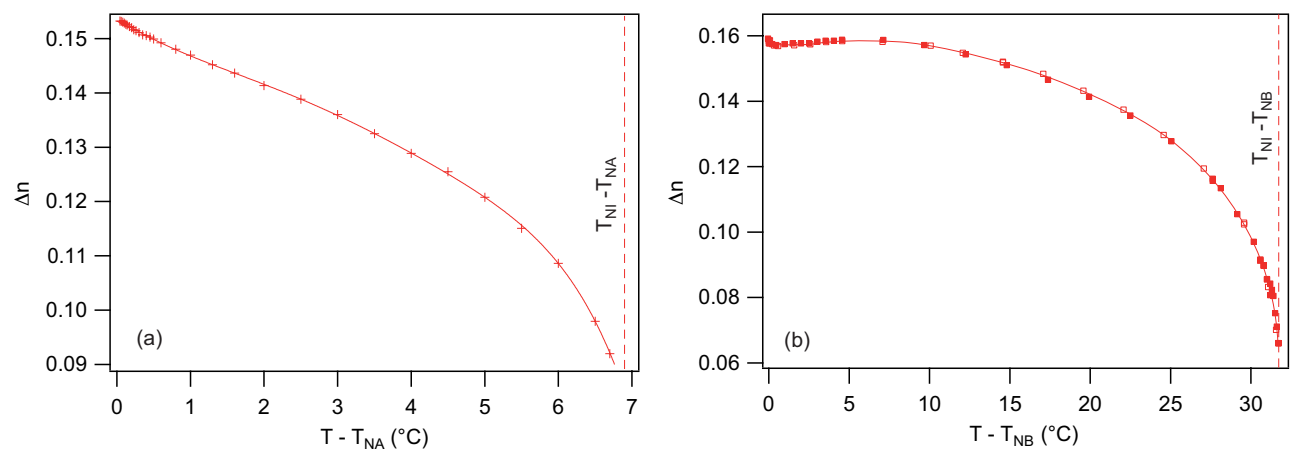

Figure 7. Birefringence $\Delta n$ as a function of temperature. (a) Pure $8 \mathrm{CB}$; (b) mixture $8 \mathrm{CB}+50 \mathrm{wt} \% \mathrm{CB} 7 \mathrm{CB}$. The two symbols $(\square, \boldsymbol{\square})$ correspond to two different samples. The solid lines are just guides for the eye.
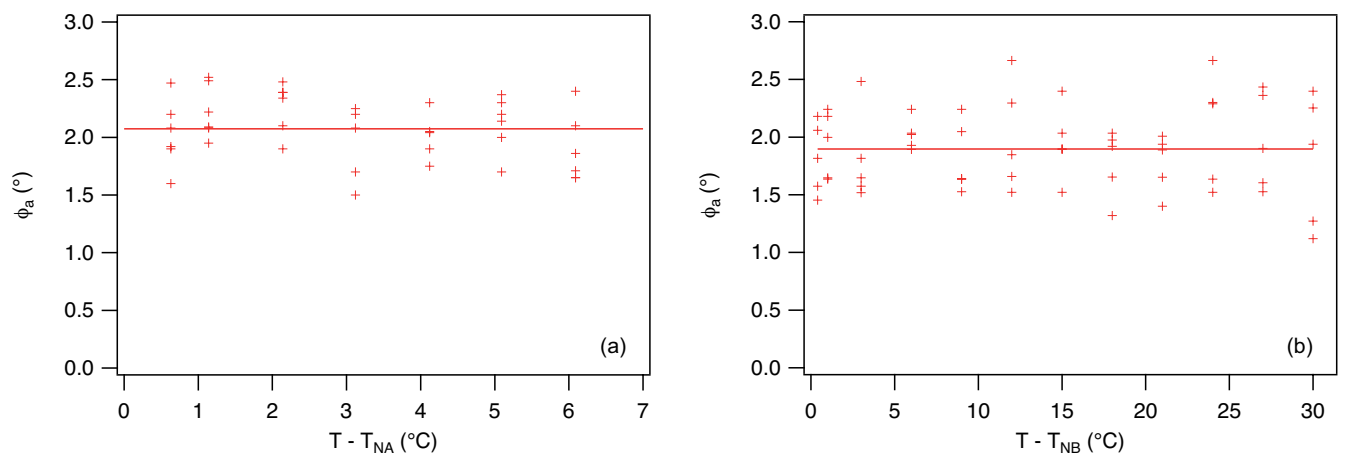

Figure 8. Pretilt angle $\phi_{a}$ as a function of temperature. (a) Pure $8 \mathrm{CB}$; (b) mixture $8 \mathrm{CB}+50 \mathrm{wt} \% \mathrm{CB} 7 \mathrm{CB}$. The solid lines give the average value of $\phi_{a}$.
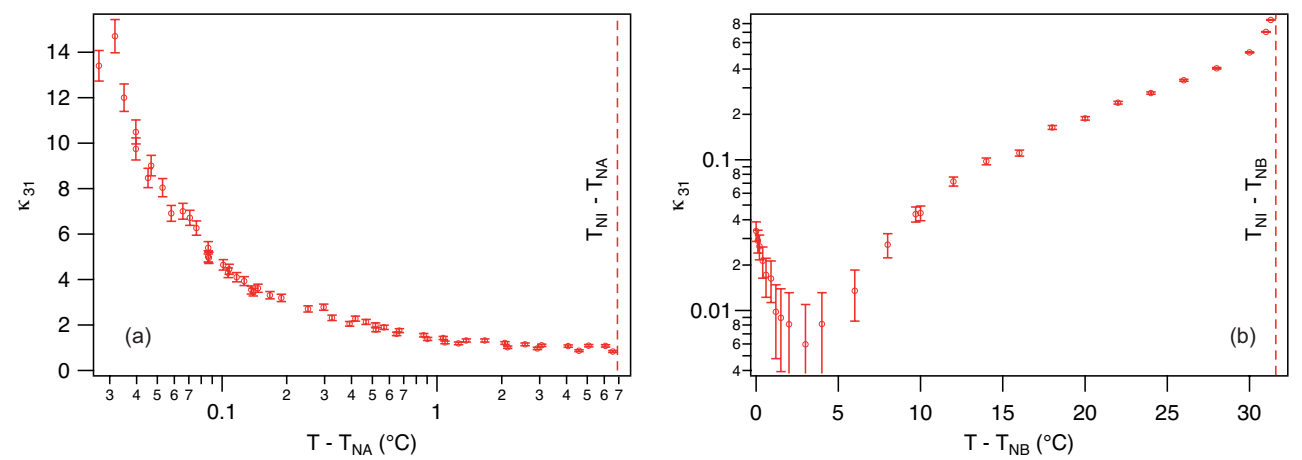

Figure 9. Elastic anisotropy $\kappa_{31}$ as a function of temperature. (a) Pure $8 \mathrm{CB}$; (b) mixture $8 \mathrm{CB}+50 \mathrm{wt} \% \mathrm{CB} 7 \mathrm{CB}$. The solid lines are just guides for the eye.

destabilized at $T_{N A}$ by forming transverse modulations which are the precursors of the focal conic domains of the smectic phase [4]. In practice, we took for $T_{N A}$ the temperature at which these transverse modulations appeared in the core of the dislocations of the banded texture (Fig. 5b). In practice this texture forms in a very narrow interval of temperature above $T_{N A}$, which depends on the sample thickness [23]. In a $25 \mu$ m-thick sample of $8 \mathrm{CB}$ this interval of temperature is of the order of $0.02^{\circ} \mathrm{C}$. Needless to say that our optical method to determine $\kappa_{31}$ can no longer be applied in this interval of temperature, which is a limitation of this method. We still specify that $T_{N I}$ is here the temperature at which the nematic phase starts to melt (solidus temperature) while $T_{N B}$ is the temperature at which the twist-bend nematic domains start to nucleate in the nematic phase (liquidus temperature). These domains are shown in Fig. 6 when the temperature is close to the solidus temperature, about $0.1^{\circ}$ below the liquidus temperature. Temperatures $T_{N A}$ and $T_{N B}$ were measured with a precision of $\pm 0.02^{\circ} \mathrm{C}$.

Fig. 7 shows the temperature variations of the birefringence measured in red-light $(\lambda=632.8 \mathrm{~nm})$ 
in the two LCs. Note that our measurements in $8 \mathrm{CB}$ are in excellent agreement with those of Morris et al. [24] performed at the same wavelength.

Fig. 8 shows the results on the pretilt angle in the two liquid crystals obtained by using the values of $n_{o}$ and $\Delta n$ given above. These measurements are noisy because of the smallness of the retardation difference between the two parts of the sample (of the order of $10 \mathrm{~nm}$ ) but it confirms the value of about $2^{\circ}$ given by Nissan in its catalog. In addition, these measurements show that the pretilt is constant within the precision of our measurements. By averaging all the measurements we found $\bar{\phi}_{a}=2.07^{\circ} \pm 0.05^{\circ}$ in $8 \mathrm{CB}$ and $\bar{\phi}_{a}=1.9^{\circ} \pm 0.05^{\circ}$ in the mixture $8 \mathrm{CB} / \mathrm{CB} 7 \mathrm{CB}$.

Finally, we report in Fig. 9 our results on the elastic anisotropy $\kappa_{31}$. They were obtained by using the preceding values of $n_{o}$ and $\Delta n$ and $\phi_{a}$ in the calculations. The error bars were calculated by taking account the errors on $\Delta n, d$ and $\phi_{a}$. As expected, we observe that $\kappa_{31}$ increases when the temperature decreases in pure $8 \mathrm{CB}$ and diverges at $T_{N A}$ whereas $\kappa_{31}$ has a non-monotone behavior in the mixture $8 \mathrm{CB} / \mathrm{CB} 7 \mathrm{CB}$. More precisely $\kappa_{31}$ decreases till a minimum is reached before increasing again when the temperature decreases and tends to $T_{N B}$. This behavior was already observed by other authors in pure $\mathrm{CB} 7 \mathrm{CB}[5]$ and in mixtures of $8 \mathrm{CB} / \mathrm{CB} 7 \mathrm{CB}[6]$ and was attributed to the presence of cybotactic groups of lamellar-like $\mathrm{N}_{\mathrm{TB}}$ phase in the nematic phase [5]. The surprising point here is that our values of $\kappa_{31}$ are extremely small in the vicinity of the $\mathrm{N}_{\mathrm{TB}}$ phase, smaller than all the values reported before in similar mixtures. For this reason, we asked questions about the accuracy of our measurements and decided to measure $\kappa_{31}$ in another way for confirmation. For this purpose, we used the classical Freedericksz transition technique by capacitance measurements.

\section{Comparison with the results obtained by using the Freedericksz transition}

To test the validity of the optical method, we measured $\kappa_{31}$ and $\phi_{a}$ by using the Freedericksz transition [25] by capacitance measurements. Before giving our results we recall a few theoretical results and we describe our experimental procedure.

\subsection{Main theoretical results}

The parametric equations used to calculate the sample capacitance $C$ as a function of the rms value $V$ of the applied voltage in the dielectric regime were known for a long time [26] when the sample is perfectly planar, i.e. when $\phi_{a}=0$ on the two glass plates and the anchoring energy is large enough to be considered as infinite. In terms of the reduced quantities

$$
\kappa=\frac{K_{3}}{K_{1}}-1 \quad \text { and } \quad \gamma=\frac{\epsilon_{\|}}{\epsilon_{\perp}}-1
$$

they read

$$
\frac{V}{V_{c}}=\frac{2}{\pi} \int_{0}^{\pi / 2} \mathrm{~d} \psi \sqrt{\frac{(1+\gamma \eta)\left(1+\kappa \eta \sin ^{2} \psi\right)}{\left(1+\gamma \eta \sin ^{2} \psi\right)\left(1-\eta \sin ^{2} \psi\right)}}
$$

and

$$
\frac{C}{C_{\perp}}=\frac{2}{\pi} \frac{V_{c}}{V} \int_{0}^{\pi / 2} \mathrm{~d} \psi \sqrt{\frac{(1+\gamma \eta)\left(1+\gamma \eta \sin ^{2} \psi\right)\left(1+\kappa \eta \sin ^{2} \psi\right)}{1-\eta \sin ^{2} \psi}}
$$

Here $V_{c}=\pi \sqrt{\frac{K_{1}}{\epsilon_{0} \epsilon_{a}}}$ is the critical voltage (by denoting by $\epsilon_{a}=\epsilon_{\|}-\epsilon_{\perp}$ the dielectric anisotropy and by $\epsilon_{0}$ the vacuum permittivity) and $C_{\perp}=\frac{\epsilon_{0} \epsilon_{\perp} S}{d}$ is the sample capacitance measured below the onset of instability (by denoting by $S$ the sample surface area). The parameter $\eta=\sin ^{2} \phi_{m}$, where $\phi_{m}$ is 


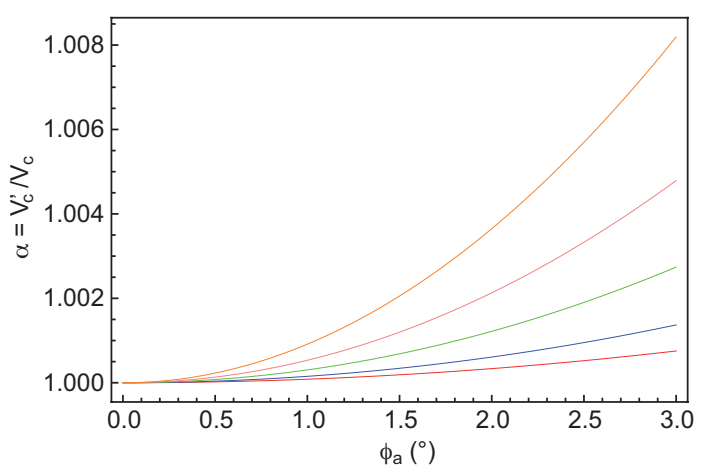

Figure 10. Multiplicative factor $k$ as a function of the pretilt angle $\phi_{a}$ calculated for different values of $\kappa$ by taken $\gamma=1$. From top to bottom $\kappa=10,5,2,0,-0.9$.

the maximum tilt angle of the director at $z=d / 2$, is given as a function of the applied voltage by solving Eq. (7). The capacitance is then calculated by using Eq. (8).

If the sample is perfectly planar, these equations can be used to fit the capacitance curves. In that case, the dielectric constant $\epsilon_{\perp}$ is obtained by measuring the capacitance $C_{\perp}$ below the onset of instability. The dielectric constant $\epsilon_{\|}$can then be obtained by noticing that, in the high voltage limit, the capacitance varies linearly with $1 / V$ and tends to $C_{\|}=\frac{\epsilon_{0} \epsilon_{\|} S}{d}$ [27]. So extrapolating the experimental curve $C(1 / V)$ at point $1 / V=0$ gives $C_{\|}$. From these measurements, the two dielectric constants $\epsilon_{\perp}$ and $\epsilon_{\|}$and the dielectric anisotropy $\epsilon_{a}$ are obtained providing the capacitance of the empty cell $C_{0}$ has been measured previously. The constant $K_{1}$ is then obtained by measuring $V_{c}$. Finally $\kappa_{31}($ or $\kappa)$ is obtained by fitting the shape of the curve with the parametric equations (7) and (8).

In practice, the situation is more complicated because our samples are not perfectly planar. Indeed, there exists a pretilt angle $\phi_{a} \neq 0$ imposed by the rubbing process, which must be taken into account in the calculations. Because this angle is well defined with respect with the rubbing direction, parallel or antiparallel samples can be prepared depending on whether the glass plates have been rubbed or not in the same direction. In these two cases the preceding formulas do no longer strictly apply and must be generalized. This point is discussed in detail in Appendix A. We just provide here the main results useful for our purpose for each type of samples. To simplify we assume that the anchoring energy is large, which can be justified a posteriori in our experiments as shown in Appendix C.

- Parallel samples

In these samples there still exists a well-defined critical voltage $V_{c}^{\prime}$ below which the capacitance is almost constant while it rapidly increases above $V_{c}^{\prime}$. This voltage is given by $V_{c}^{\prime}=\alpha V_{c}$ where $\alpha$ is a multiplicative factor given by

$$
\alpha=\frac{2}{\pi} \int_{0}^{\phi_{a}} \mathrm{~d} \phi \sqrt{\frac{\left(1+\kappa \sin ^{2} \phi\right)\left(1+\gamma \sin ^{2} \phi_{a}\right)}{\left(1+\gamma \sin ^{2} \phi\right)\left(\sin ^{2} \phi_{a}-\sin ^{2} \phi\right)}}
$$

In practice $\alpha$ is always very close to 1 as we can see in Fig. 10.

Below the onset of instability, the capacitance is no longer constant but slightly increases when the voltage increases. In this regime, the solution $\phi(z)$ is an odd function with respect $z=d / 2$, that is $\phi(d-z)=-\phi(z)$, so that $\phi(d / 2)=0$ at all voltages below $V_{c}^{\prime}$. The capacitance also slightly increases when the voltage increases because the molecules tilt under the action of the electric field close to the plates. In this regime the capacitance tends to a value slightly different from $C_{\perp}$ when $V \rightarrow 0$. This value is independent of $\kappa$ (this can be easily shown by using Eqs. (A15) and (A16) of Appendix A) and given by

$$
C_{\perp}^{0}=C_{\perp}\left(1+\frac{\gamma}{3} \phi_{a}^{2}\right)
$$



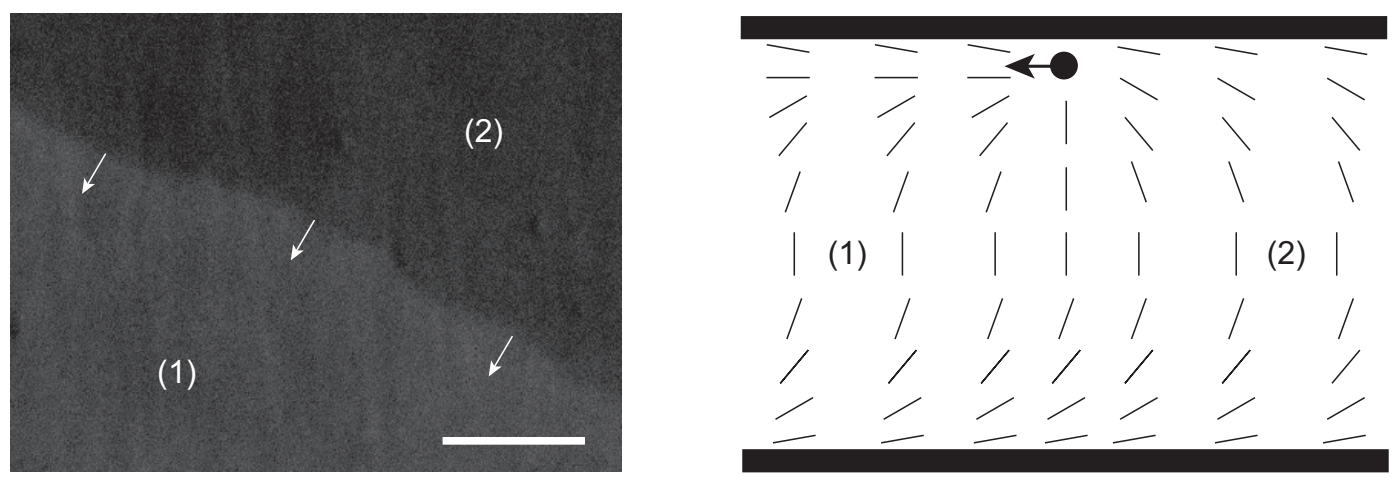

Figure 11. $\pi$-wall domain (2) invading a normal domain (1) in a parallel sample of $8 \mathrm{CB}$ of thickness $25 \mu \mathrm{m}$ by propagation of a $+1 / 2$ surface disclination line. This transformation produces when the applied voltage is larger than 15 Vrms typically. The photo is taken between crossed polarizers and the white bar is $40 \mu \mathrm{m}$ long. The contrast has been strongly enhanced to better visualize the two regions. The drawing shows the surface disclination line separating the two regions.

With $\phi_{a}=2^{\circ}$, one finds $C_{\perp}^{0}=1.0004 C_{\perp}$ which is indeed a very small correction.

More interesting is the shape of the curve above the onset of instability. In this regime, it can be shown that formulas (7) and (8) remain applicable. This result is important because it shows that parallel samples behave as perfect samples to a very good approximation, independently of the value of $\phi_{a}$ (providing $\phi_{a} \ll 1$ ). For this reason we used parallel samples to measure $\kappa_{31}$.

However, parallel samples often destabilize at large voltage by forming a $\pi$-wall within the sample thickness. This phenomenon was known for a long time [28] and is shown in Fig. 11. In this regime, the previous formulas are inapplicable, but the capacitance curve can still be calculated by using other parametric equations that are given in Appendix A. From these formulas, it can be shown that the capacitance still varies linearly with $1 / V$ and tends to $C_{\|}$in the limit $1 / V=0$. In this respect, the parallel samples behave again as perfect planar samples, even when a $\pi$-wall has formed.

For all reasons we used parallel samples to measure the dielectric and elastic constants.

- Antiparallel samples

The behavior of antiparallel samples is very different. This is due to the fact that the director is never perpendicular to the electric field in the center of the sample so that the sample immediately destabilizes when an electric field is applied. For this reason the capacitance curves round in the vicinity of $V_{c}$ and the onset of instability disappears. This effect strongly depends on the pretilt angle and can be used to measure it $[19,20]$. In that case, the calculations show that the capacitance is still given by Eqs (7) and (8) on condition of replacing the lower limit of all the integrals by

$$
\psi_{0}=\operatorname{asin}\left(\frac{\sin \phi_{a}}{\sqrt{\eta}}\right)
$$

instead of 0 . The capacitance of antiparallel samples tend to

$$
C_{\perp}^{0}=C_{\perp}\left(1+\gamma \sin ^{2} \phi_{a}\right)
$$

when $V \rightarrow 0$, which turns out to be again a very small correction to $C_{\perp}$ when $\phi_{a}$ is small. Finally, the capacitance varies linearly with $1 / V$ and tends to $C_{\|}$in the limit $1 / V=0$ as in a perfect planar sample.

\section{$5.2 \quad$ Experimental procedure}

In practice, we made parallel and antiparallel samples of the two liquid crystals studied. For each sample, the capacitance $C_{0}$ of the empty cell was first measured. Then the cell was filled with the LC in the isotropic phase and placed in the precision oven described above. The curves of capacitance 


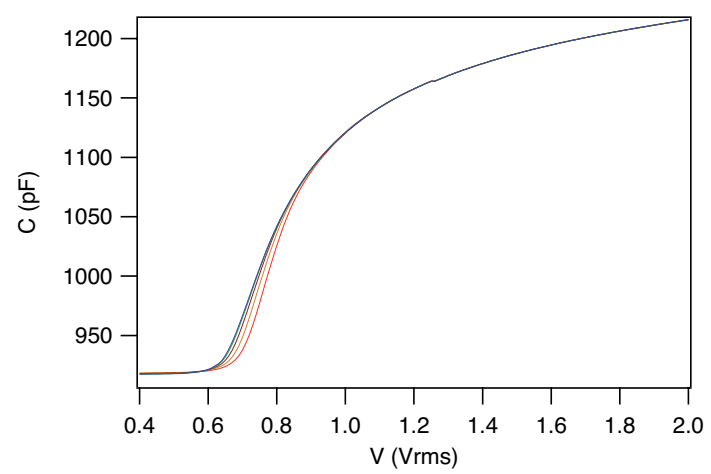

Figure 12. Evolution as a function of $\Delta t$ of the curve of capacitance measured in an antiparallel sample of the mixture $8 \mathrm{CB} / \mathrm{CB} 7 \mathrm{CB}$ at $T=62.1^{\circ} \mathrm{C}\left(T_{N I}-T=0.5^{\circ} \mathrm{C}\right)$. From right to left, $\Delta t=1,2,4,8$ and $12 \mathrm{~s}$. These recordings show that a time delay of $12 \mathrm{~s}$ minimum is required to measure a curve at equilibrium.

of each sample was then measured at small and large voltages at different temperatures. In the case of the antiparallel samples the two curves were measured one after the other before each change of temperature. This was possible because the samples do not destabilize at high voltage. In the case of the parallel samples this was not possible because of the formation of the $\pi$-wall at large voltage. For this reason, we first recorded the curves at small voltage as a function of temperature and then the curves at large voltages after ensuring that the $\pi$-wall was formed. All measurements were done with a LCR meter HP 4284A. With this apparatus the voltage can be changed between 0 and 20 Vrms. The measurements at low voltages were performed between $10 \mathrm{mV}$ and $2 \mathrm{~V}$ with increments of 10 $\mathrm{mV}$ (200 points) in the parallel samples and between $10 \mathrm{mV}$ and the value of $V_{c}$ in the antiparallel samples by taking 60 points. The measurements at large voltages were performed between 5 and $20 \mathrm{~V}$ with increments of $0.1 \mathrm{~V}$ (150 points). All the measurements were performed in the dielectric regime. With $8 \mathrm{CB}$, we measured a charge relaxation frequency $f_{c}$ of the order of $200 \mathrm{~Hz}$ and our measurements were done at $5 \mathrm{kHz}$. With the $8 \mathrm{CB} / \mathrm{CB} 7 \mathrm{CB}$ mixture, $f_{c}$ was higher, of the order of $1.5 \mathrm{kHz}$, and our measurements were done at $50 \mathrm{kHz}$. A crucial point was to record equilibrium curves. For this reason, it was important to wait long enough between each of the measuring points. To determine the optimal time delay $\Delta t$ between each measurement, we recorded several curves at the same temperature with different $\Delta t$. The optimal delay was obtained when all the curves superposed. It can change with temperature and it must be determined seriously, in particular when a phase transition is approached. In the case of $8 \mathrm{CB}$, a delay of $5 \mathrm{~s}$ was enough when $T-T_{N A}>1^{\circ} \mathrm{C}$. By contrast, this delay must be increased in the critical regime and may be as long as $30 \mathrm{~s}$ when $T-T_{N A}$ is of the order of $0.1^{\circ} \mathrm{C}$. This problem also arose with the mixture $8 \mathrm{CB} / \mathrm{CB} 7 \mathrm{CB}$ because of its large rotational viscosity (ranging typically between 0.2 Pa.s at $T_{N I}$ and more than 2 Pa.s close to $\left.T_{N B}\right)$. With this mixture, we used a delay of $20 \mathrm{~s}$ in general and up to $30 \mathrm{~s}$ close to the $\mathrm{N}_{\mathrm{TB}}$ phase. One example of curves recorded with different $\delta t$ close to the clearing point of this mixture is shown in Fig. 11. This discussion shows that experiments are very long with this technique, which can cause problems if the samples degrade or crystallize as it has been observed many times in the mixture with CB7CB.

\subsection{Experimental results}

Examples of curves recorded at low and high voltages in parallel and antiparallel samples are shown in Figs. 13 and 14. From these curves, the capacitance $C_{\perp}^{0}$ was determined by extrapolating to 0 the curves $\mathrm{C}(\mathrm{V})$ measured at low voltages (Fig. 13). The capacitance $C_{\|}$was determined by extrapolating to 0 the linear fits of the curves $C(1 / V)$ measured at large voltages (Fig. 14). The capacitance $C_{\perp}$ was then calculated by taking $\phi_{a}=2^{\circ}$ in Eqs. (10) and (12) and the dielectric constants $\epsilon_{\perp}$ and $\epsilon_{\|}$were calculated by dividing $C_{\perp}$ and $C_{\|}$by the capacitance $C_{0}$ of the empty cells. From these measurements the dielectric anisotropy $\epsilon_{a}$ and the factor $\gamma$ were obtained.

The next step was to fit the curves measured with parallel samples with the parametric equations 

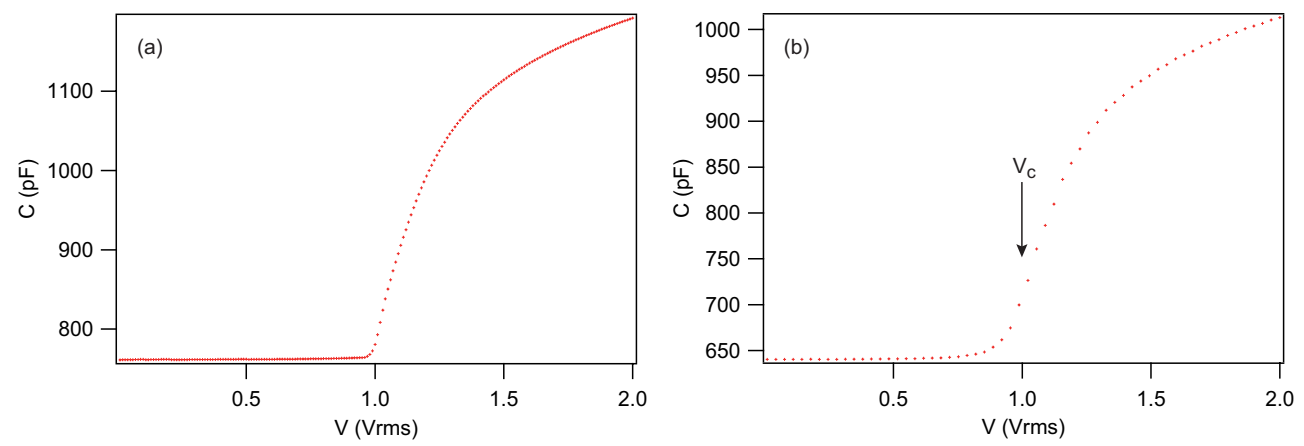

Figure 13. Capacitance as of function of the applied voltage in a parallel sample (a) and in an antiparallel sample (b) of the mixture $8 \mathrm{CB} / \mathrm{CB} 7 \mathrm{CB}$. Curves measured at $T-T_{N B}=22.6^{\circ} \mathrm{C}$.
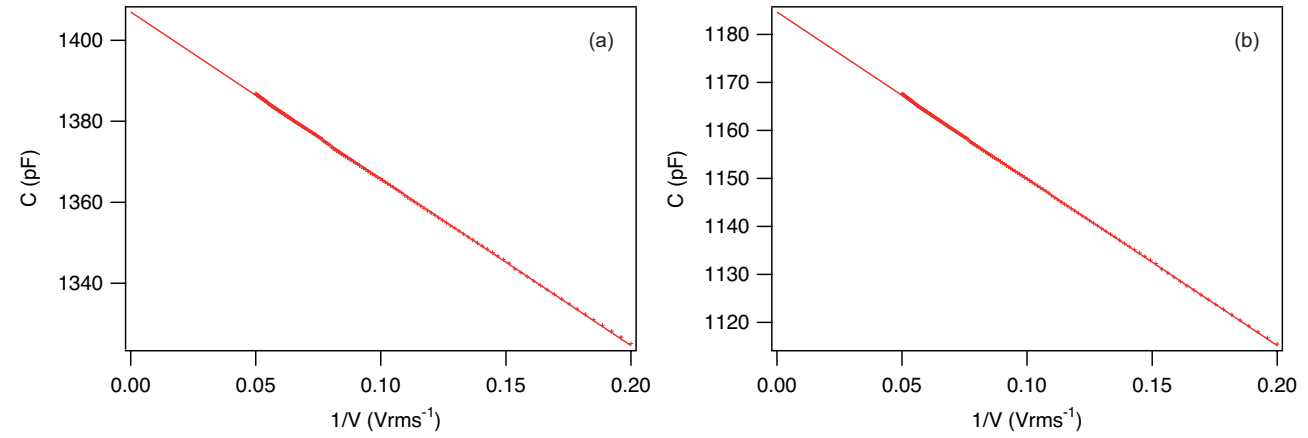

Figure 14. Capacitance as of function of the reciprocal of the applied voltage in a parallel sample in which a $\pi$-wall has formed (a) and in an antiparallel sample (b). Mixture $8 \mathrm{CB} / \mathrm{CB} 7 \mathrm{CB}$. Curves measured at $T-T_{N B}=22.6^{\circ} \mathrm{C}$. The solid lines are the best fits with a linear function.
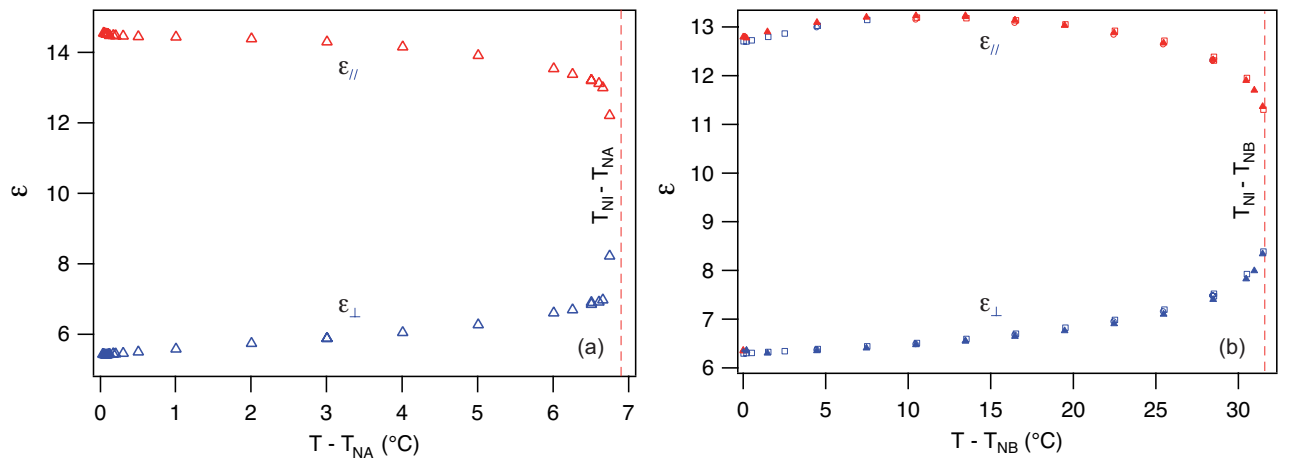

Figure 15. Dielectric constants measured in $8 \mathrm{CB}$ (a) and the mixture $8 \mathrm{CB} / \mathrm{CB} 7 \mathrm{CB}$ (b). The solid symbols correspond to measurements with parallel samples and the open symbols correspond to measurements with antiparallel samples.
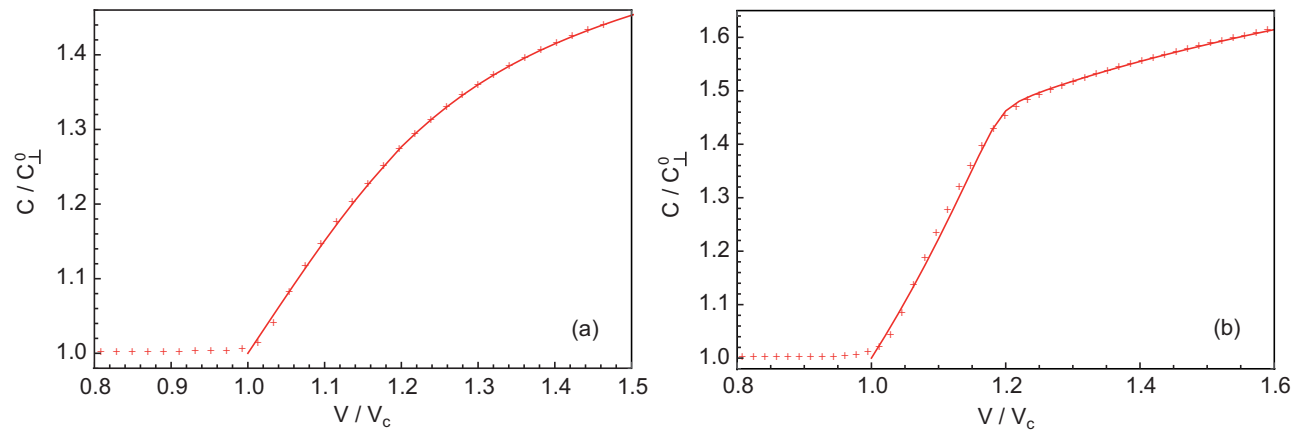

Figure 16. Two examples of fit (solid lines) of the reduced capacitance data (crosses) obtained with a parallel sample of thickness $d=25 \mu \mathrm{m}$ of the mixture $8 \mathrm{CB} / \mathrm{CB} 7 \mathrm{CB}$ at $T-T_{N B}=22.47^{\circ} \mathrm{C}$ (a) and $T-T_{N B}=4.47^{\circ} \mathrm{C}$. In these two graphs, half the experimental points have been reported for more clarity. In (a) $\kappa=-0.768$ and in (b) $\kappa=-0.99$. 

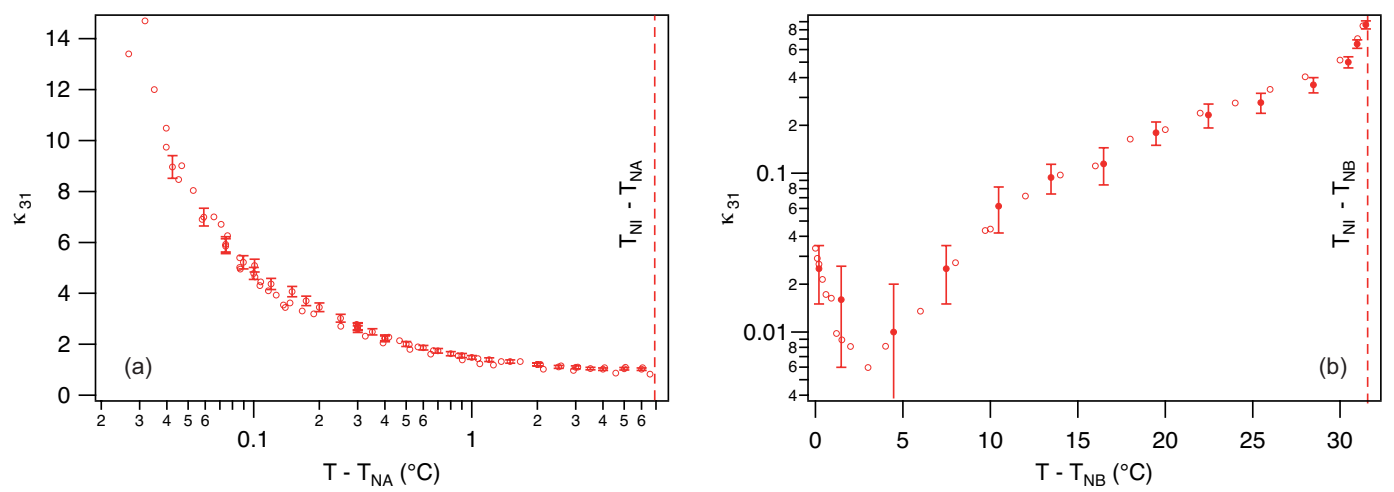

Figure 17. Comparison between the two methods. The open symbols have been measured with the optical method and the solid symbols with the capacitive method. (a) $8 \mathrm{CB}$; (b) mixture $8 \mathrm{CB} / \mathrm{CB} 7 \mathrm{CB}$.
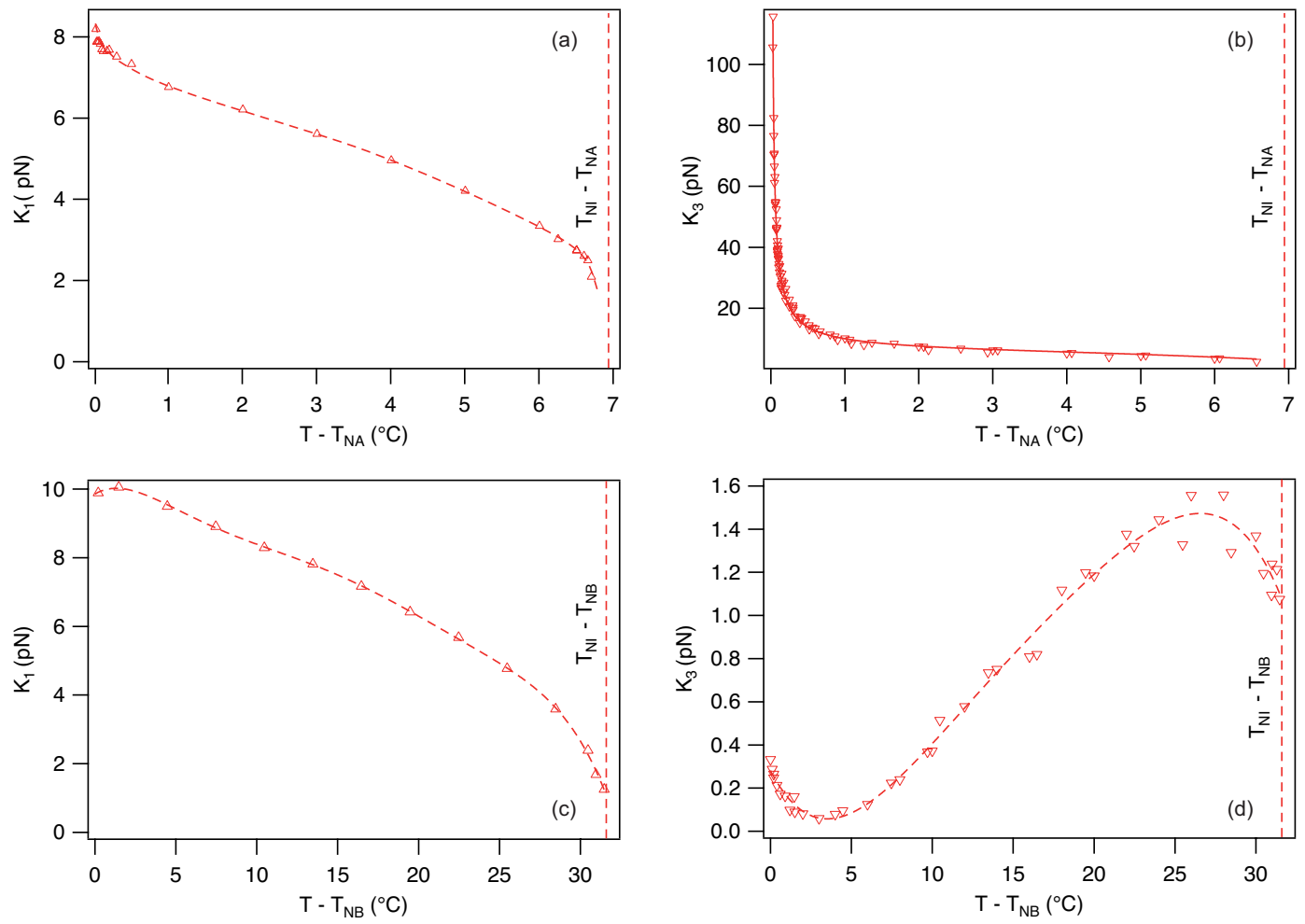

Figure 18. Elastic constants in $8 \mathrm{CB}(\mathrm{a}, \mathrm{b})$ and the mixture $8 \mathrm{CB} / \mathrm{CB} 7 \mathrm{CB}(\mathrm{c}, \mathrm{d})$. The solid line in (b) is the best fit of the data with Eq. (17). The other dashed lines are just guides for the eye.

(7) and (8). Since $\gamma$ was already known, the number of fit parameters was reduced to 2 ; that is $V_{c}$ and $\kappa\left(\right.$ or $\left.\kappa_{31}\right)$. The fits were done using Mathematica by minimizing the shift between the theoretical and experimental curves

$$
\Delta\left(V_{c}, \kappa\right)=\sqrt{\frac{1}{N} \sum_{i=1}^{N}\left[C_{\exp }(i)-C_{\text {theo }}(i)\right]^{2}}
$$

In this expression, $i$ is the number of the point measured at voltage $V(i), N$ is the total number of points measured above the onset of instability, $C_{\exp }(i)$ is the capacitance measured at $V(i)$ and $C_{\text {theo }}(i)$ is the capacitance calculated at voltage $V(i)$ by using Eqs. (7) and (8). Note that the 


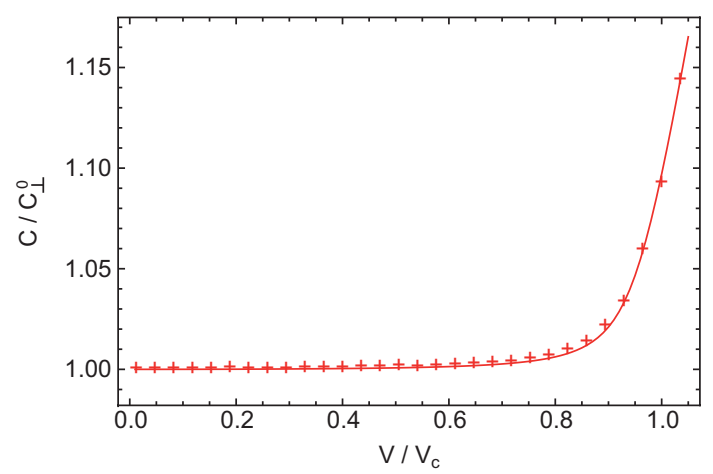

Figure 19. Example of fit (solid line) of the reduced capacitance data (crosses) obtained with an antiparallel sample of thickness $d=25 \mu \mathrm{m}$ of the mixture $8 \mathrm{CB} / \mathrm{CB} 7 \mathrm{CB}$ at $T-T_{N B}=22.47^{\circ} \mathrm{C}$.

integrals in these equations were calculated by using a very useful formula given in Ref. [24]:

$$
\int_{0}^{\pi / 2} \frac{f(\psi) \mathrm{d} \psi}{\sqrt{1-\eta \sin ^{2} \psi}}=e^{\alpha / 2} \int_{0}^{\pi / 2} \mathrm{~d} \psi \frac{f(\psi)-f(\pi / 2)}{\sqrt{1+\left(e^{\alpha}-1\right) \cos ^{2} \psi}}+f(\pi / 2) K\left(1-e^{-\alpha}\right)
$$

where $\eta=1-e^{-\alpha}$ and $K(\eta)$ denotes the complete elliptic integral of the first kind

$$
K(\eta)=\int_{0}^{\pi / 2} \frac{\mathrm{d} \psi}{\sqrt{1-\eta \sin ^{2} \psi}}
$$

easy to calculate when $\eta \rightarrow 1$ by using the series expansion

$$
K(1-\epsilon)=\left(2 \log (2)-\frac{\log (\epsilon)}{2}\right)+\frac{1}{8} \epsilon(-\log (\epsilon)-2+4 \log (2))+O\left(\epsilon^{2}\right)
$$

From the fits of the experimental curves (two typical examples are shown in Fig. 16), values of $V_{c}$ and $\kappa\left(\right.$ or $\left.\kappa_{31}\right)$ were determined. In Fig. 17, the values of $\kappa_{31}$ found in this way are compared with the values obtained using the optical method described in the previous section. The agreement is good in the two liquid crystals, which confirms the validity of the optical method. This good agreement also gave us confidence that flexoelectric effects were screened out in our experiments and could be neglected. This point is justified in Appendix B.

For completeness we also calculated $K_{1}$ and $K_{3}$. Note that the values of $K_{1}$ used to calculate $K_{3}$ from the optical values of $\kappa_{31}$ were interpolated from polynomial fits of the curves $K_{1}(T)$. The results are shown in Fig. 18. For 8CB, we also determined the critical exponent of the divergent part of $K_{3}$ by fitting the curve $K_{3}(T)$ with the equation [24]

$$
K_{3}=a \Delta n^{2}+\frac{b}{\left(T-T_{N A}\right)^{x}}
$$

In this equation the first term proportional to $\Delta n^{2}$ represents the background nematic contribution to $K_{3}$. The best fit with Igor gave $x=0.948 \pm 0.035$. This exponent is compatible with the value of the order of 1 found by Morris et al. [24] and others [29-31] by using the Freedericksz transition, but is larger than the value of about 0.7 given by light scattering [32, 33] and X-rays experiments [34, 35] and also found by Scudieri by using an optical method similar to ours in cylindrical capillaries [11].

Finally, we determined the pretilt angle $\phi_{a}$ by fitting the curves of capacitance measured in antiparallel samples between $0.01 \mathrm{Vrms}$ and the critical voltage $V_{c}$. As the parameters $V_{c}, \gamma$ and $\kappa$ were already known, the only fit parameter was the pretilt angle $\phi_{a}$. Fig. 19 shows an example of fit in a sample of the mixture $8 \mathrm{CB} / \mathrm{CB} 7 \mathrm{CB}$. From these fits, $\phi_{a}$ was determined as a function of temperature in both liquid crystals. The results are shown in Fig. 20 and show that the pretilt angle is close 

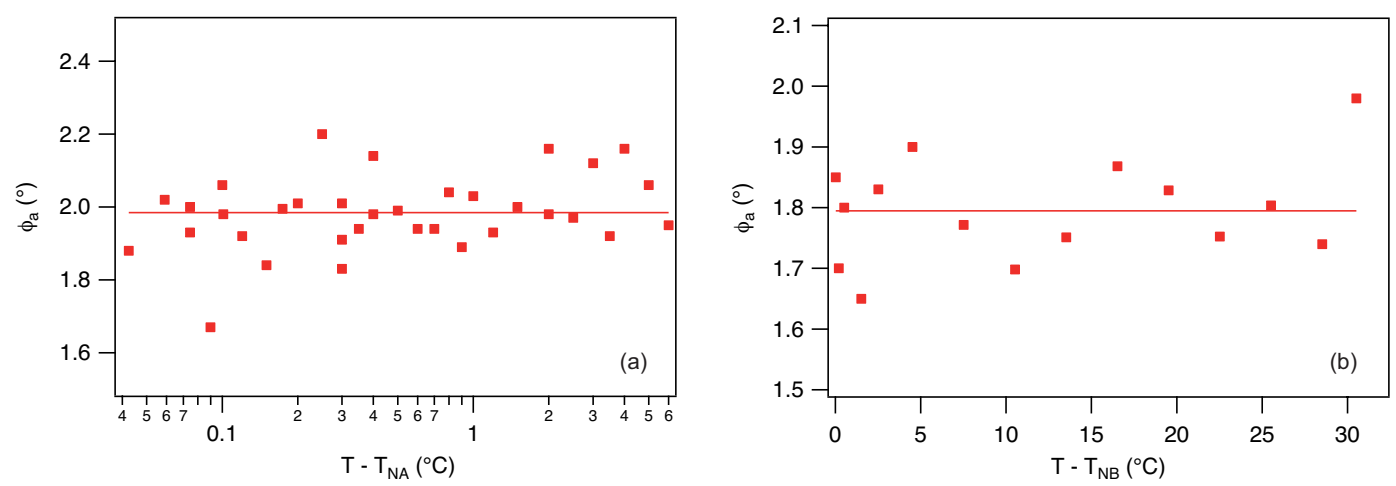

Figure 20. Pretilt angle measured with the capacitive method in $8 \mathrm{CB}$ (a) and in the mixture 8CB/CB7CB (b).

to $2^{\circ}$ in $8 \mathrm{CB}$ and a bit smaller, close to $1.8^{\circ}$, in the mixture $8 \mathrm{CB} / \mathrm{CB} 7 \mathrm{CB}$. These measurements are consistent with the orders of magnitude obtained by using the optical method described in the previous section.

\section{Conclusion}

In conclusion, we developed an optical method to measure the elastic anisotropy of nematic liquid crystals. This method turned out to be very accurate provided that the sample thickness and the indices of the LC are known very precisely. The knowledge of the pretilt angle $\phi_{a}$ is also very important. In practice, $\phi_{a}$ is difficult to measure so that, in our experiments, the main error on $\kappa_{31}$ came from the uncertainties on this angle. For this reason, the optical method would be much more precise if we had used a surface treatment that gave a perfect planar anchoring. Such surface treatments exist but we did not have them. We can cite, for instance, the surface treatment by deposition under oblique incidence of a layer of $\mathrm{SiO}$, at least under certain conditions [36], or certain treatments with photopolymers [12]. This method also requires to have an adequate polymer to obtain a strong homeotropic anchoring, which was the case with $8 \mathrm{CB}$ and the mixture $8 \mathrm{CB}-\mathrm{CB} 7 \mathrm{CB}$ used in this paper. Unfortunately this not always possible, in particular with NTB-forming dimers in which obtaining the homeotropic alignment is often an extremely difficult task.

The optical method has also numerous advantages with respect to the capacitive method. It is local, and for this reason much less sensible to temperature or chemical gradients than the capacitive method. This is a considerable advantage to study the critical behavior of $\kappa_{31}$ near phase transitions such as the nematic-to-smectic A or nematic-to- $\mathrm{N}_{\mathrm{TB}}$ phase transitions. Another advantage of the optical method is that the measurements are much easier to analyze than with the capacitive method. There is indeed no need to solve complicated parametric equations to fit the data. The optical method is also much faster to implement than the capacitive method which becomes a substantial advantage when the samples degrade or crystallize as it was the case with the mixture with CB7CB.

We underline that flexoelectric effects are completely negligible in these experiments as long as the Debye length is much smaller than the sample thickness. This was indeed the case in our experiments. In particular, we believe that using the Freedericksz transition to measure $e^{\star}$, as suggested by Brown and Mottram in Ref. [7], is very difficult in as much as the Lcs are in practice often impure.

Finally, we mention the possibility to measure the pretilt angle and the anchoring energy by measuring as a function of the sample thickness the behavior at large voltage of the curves $C(1 / V)$ measured in parallel or antiparallel samples. 


\section{Acknowledgments}

One of the authors (PO) warmly thanks Félix Bunel for his help with the LabView programs and Pawel Pieranski for useful comments.

\section{References}

[1] Oswald P, Pieranski P. Nematic and cholesteric liquid crystals: concepts and physical properties illustrated by experiments. Boca Raton: CRC press; 2005.

[2] De Gennes PG, Prost J. The physics of liquid crystals. Oxford: Clarendon Press; 1993.

[3] Jákli A, Lavrentovich OD, Selinger JV. Physics of liquid crystals of bent-shaped molecules. Rev Mod Phys. 2018;90(4):045004.

[4] Oswald P, Pieranski P. Smectic and columnar liquid crystals: concepts and physical properties illustrated by experiments. Boca Raton: CRC press; 2006.

[5] Babakhanova G, Parsouzi Z, Paladugu S, et al. Elastic and viscous properties of the nematic dimer CB7CB. Phys Rev E. 2017;96(6):062704.

[6] Aouini A, Nobili M, Chauveau E, et al. Chemical-physical characterization of a binary mixture of a twist bend nematic liquid crystal with a smectogen. Crystals. 2020;10(12):1110.

[7] Brown C, Mottram N. Influence of flexoelectricity above the nematic fréedericksz transition. Phys Rev E. 2003;68(3):031702.

[8] Lee JB, Pelcovits RA, Meyer RB. Role of electrostatics in the texture of islands in free-standing ferroelectric liquid crystal films. Phys Rev E. 2007;75(5):051701.

[9] Meyer RB. Piezoelectric effects in liquid crystals. Phys Rev Lett. 1969;22(18):918.

[10] Scudieri F. Cylindrically aligned liquid crystals: an interferometric analysis. Appl Opt. 1979;18(9):14551459.

[11] Scudieri F. Optical measurement of KB/KS divergence in nematics. Opt Commun. 1981;37(1):37-39.

[12] Ishihara S, Mizusaki M. Alignment control technology of liquid crystal molecules. J Soc Inf Disp. 2020; 28(1):44-74.

[13] Baur G, Wittwer V, Berreman D. Determination of the tilt angles at surfaces of substrates in liquid crystal cells. Phys Lett A. 1976;56(2):142-144.

[14] Scheffer T, Nehring J. Accurate determination of liquid-crystal tilt bias angles. J Appl Phys. 1977; 48(5):1783-1792.

[15] Kosmowski B, Becker K, Cremers R, Mlynski D. Tilt bias angle measurement with improved sensitivity. Mol Cryst Liq Cryst. 1981;72(1):17-25.

[16] Riviere D, Levy Y, Guyon E. Determination of anchoring energies from surface tilt angle measurements in a nematic liquid crystal. J Phys Lett (Paris). 1979;40(10):215-218.

[17] Faetti S, Mutinati G. An improved reflectometric method to measure the azimuthal anchoring energy of nematic liquid crystals. Eur Phys J E. 2003;10(3):265-279.

[18] Chen KH, Chang WY, Chen JH. Measurement of the pretilt angle and the cell gap of nematic liquid crystal cells by heterodyne interferometry. Opt Express. 2009;17(16):14143-14149.

[19] Meyerhofer D. Field induced distortions of a liquid crystal with various surface alignments. Phys Lett A. $1975 ; 51(7): 407-408$.

[20] Toko Y, Akahane T. Evaluation of pretilt angle and polar anchoring strength of amorphous alignment liquid crystal display from capacitance versus applied voltage measurement. Mol Cryst Liq Cryst Section A. 2001;368(1):469-481.

[21] Dequidt A, Oswald P. Zigzag instability of a $\chi$ disclination line in a cholesteric liquid crystal. Eur Phys J E. 2006;19(4):489-500.

[22] Cladis P, Torza S. Growth of a smectic a from a bent nematic phase and the smectic light valve. J Appl Phys. 1975;46(2):584-599.

[23] Zappone B, Mamuk AE, Gryn I, et al. Analogy between periodic patterns in thin smectic liquid crystal films and the intermediate state of superconductors. PNAS. 2020;117(30):17643-17649.

[24] Morris SW, Palffy-Muhoray P, Balzarini D. Measurements of the bend and splay elastic constants of octyl-cyanobiphenyl. Mol Cryst Liq Cryst. 1986;139(3-4):263-280.

[25] Fréedericksz V, Zolina V. Forces causing the orientation of an anisotropic liquid. Trans Faraday Soc. 1933;29(140):919-930. 
[26] Deuling HJ. Deformation of nematic liquid crystals in an electric field. Mol Cryst Liq Cryst. 1972; 19(2):123-131.

[27] Uchida T, Takahashi Y. New method to determine elastic constants of nematic liquid crystal from cv curve. Mol Cryst Liq Cryst. 1981;72(4):133-137.

[28] Dozov I, Martinot-Lagarde P. First-order breaking transition of tilted nematic anchoring. Phys Rev E. 1998;58(6):7442.

[29] Cheung L, Meyer R. Pretransitional anomaly in the bend elastic constant for a nematic to smectic A transition. Phys Lett A. 1973;43(3):261-262.

[30] Jánossy I, Bata L. Study of elastic properties near a nematic-smectic-A transition. Acta Phys Pol. 1978; A54(5):643.

[31] DasGupta S, Roy SK. The bend elastic constant in a mixture of 4, 4?-n-octyl-cyanobiphenyl and biphenyl. Phys Lett A. 2001;288(5-6):323-328.

[32] Sprunt S, Solomon L, Litster J. Equality of x-ray and light scattering measurements of coherence lengths at the nematic-smectic-a phase transition. Phys Rev Lett. 1984;53(20):1923.

[33] Birecki H, Litster J. Director bend mode behavior near a nematic to smectic-a phase-transition. Mol Cryst Liq Cryst. 1977;42(1-3):1043-1051.

[34] Ocko B, Birgeneau R, Litster J, et al. Critical and tricritical behavior at the nematic to smectic-a transition. Phys Rev Lett. 1984;52(3):208.

[35] Garland C, Meichle M, Ocko B, et al. Critical behavior at the nematic-smectic-a transition in butyloxybenzylidene heptylaniline (4o. 7). Phys Rev A. 1983;27(6):3234.

[36] Jerome B, Pieranski P, Boix M. Bistable anchoring of nematics on sio films. EPL. 1988;5(8):693.

[37] Gruler H, Scheffer TJ, Meier G. Elastic constants of nematic liquid crystals: I. theory of the normal deformation. Z Naturforsch A. 1972;27(6):966-976.

[38] Varanytsia A, Chien LC. Giant flexoelectro-optic effect with liquid crystal dimer cb7cb. Sci Rep. 2017; $7(1): 1-7$.

[39] Krishnamurthy KS, Palakurthy NB, Yelamaggad CV. Confined electroconvective and flexoelectric instabilities deep in the freedericksz state of nematic CB7CB. J Phys Chem B. 2017;121(21):5447-5454.

[40] Buka A, Éber N. Flexoelectricity in liquid crystals: theory, experiments and applications. Singapour: World Scientific; 2013.

[41] Bazant MZ, Thornton K, Ajdari A. Diffuse-charge dynamics in electrochemical systems. Phys Rev E. 2004;70(2):021506.

[42] Khazimullin MV, Lebedev YA. Influence of dielectric layers on estimates of diffusion coefficients and concentrations of ions from impedance spectroscopy. Phys Rev E. 2019;100(6):062601.

[43] Deuling H. The piezo-electric effect in nematic layers. Mol Cryst Liq Cryst. 1974;26(3-4):281-284.

[44] Dozov I, Barbero G, Palierne J, et al. Nonlocal electric field and large distortions in nematic liquid crystals. EPL. 1986;1(11):563.

[45] Palierne J. Elasticlike contribution of electric origin to the distortion free energy of nematics. Phys Rev Lett. 1986;56(11):1160.

[46] Rapini A, Papoular M. Distorsion d'une lamelle nématique sous champ magnétique conditions d'ancrage aux parois. J Phys Coll (Paris). 1969;30(C4):C4-54.

[47] Faetti S, Marianelli P. Strong azimuthal anchoring energy at a nematic-polyimide interface. Phys Rev E. 2005;72(5):051708.

\section{Appendix A. Capacitance in parallel and antiparallel samples: role of the pretilt angle}

The calculation of the capacitance of a planar nematic slab has already been considered by several authors when $\phi_{a}=0[26,37]$. These calculations can be easily extended to antiparallel samples in which $\phi_{a} \neq 0[20]$. By contrast, the calculations are more complex for parallel samples because the solution is no longer symmetric with respect to the mid-plane $z=d / 2$ above the onset of instability. For this reason we shall consider separately these two cases. In the following we neglect the flexoelectric effects and we assume that the anchoring is infinitely strong. 
In all cases, the capacitance is obtained from formula

$$
C=\frac{D_{z} S}{V_{a}}
$$

by minimizing the total free energy

$$
F=\int_{0}^{d} \mathrm{~d} z\left\{\frac{1}{2} K(\phi) \phi^{\prime 2}-\frac{1}{2} D_{z} E\right\}
$$

under the assumption that in the dielectric regime -i.e. at large frequency with respect to the charge relaxation frequency $f_{c}$ - the net free charge $\rho=0$. As a consequence $\vec{\nabla} \cdot \vec{D}=0$ and $D_{z}$ is a function of $t$ only. In these equations, $S$ is surface area of the sample, $V_{a}=\int_{0}^{d} E \mathrm{~d} z=\sqrt{2} V \cos (\omega t)$ is the applied voltage, and $D_{z}$ is the vertical component of the electric displacement field $\vec{D}$ given by

$$
D_{z}=\epsilon_{0} \epsilon(\phi) E
$$

where $\epsilon(\phi)=\epsilon_{\perp} \cos ^{2} \phi+\epsilon_{\|} \sin ^{2} \phi$. As $D_{z}$ is a function of $t$ only, we can write $V_{a}=\left(D_{z} / \epsilon_{0}\right) \int_{0}^{d} \epsilon(\phi)^{-1} \mathrm{~d} z$ or equivalently

$$
D_{z}=\frac{\epsilon_{0} V_{a}}{\int_{0}^{d} \epsilon(\phi)^{-1} \mathrm{~d} z}
$$

which gives after replacing $D_{z}$ and $E$ by their expressions as a function of $V_{a}$ in Eq. (A2)

$$
F=\int_{0}^{d} \frac{1}{2} K(\phi) \phi^{\prime 2} \mathrm{~d} z-\frac{1}{2} \epsilon_{0} \frac{V_{a}^{2}}{\int_{0}^{d} \epsilon(\phi)^{-1} \mathrm{~d} z}
$$

Minimizing $F$ with respect to $\phi$ gives

$$
\left(1+\kappa \sin ^{2} \phi\right) \phi^{\prime \prime}+\kappa \sin \phi \cos \phi \phi^{2}+\frac{\epsilon_{a} D_{z}^{2}}{K_{1} \epsilon_{0} \epsilon_{\perp}} \frac{\sin \phi \cos \phi}{1+\gamma \sin ^{2} \phi}=0
$$

This equation has the first integral

$$
\left(1+\kappa \sin ^{2} \phi\right) \phi^{\prime 2}=\frac{D_{z}^{2}}{K_{1} \epsilon_{0} \epsilon_{\perp}} \frac{\sin ^{2} \phi_{m}-\sin ^{2} \phi}{\left(1+\gamma \sin ^{2} \phi\right)\left(1+\gamma \sin ^{2} \phi_{m}\right)}
$$

where $\phi=\phi_{m}$ is the maximum tilt angle. Equations (A1), (A4) and (A7) are the three basic equations of the problem. Note that in these equations $\phi, C$ and $D_{z}$ are function of time since an AC voltage is used. In practice, the frequency $\omega$ of the applied voltage is much larger than the viscous relaxation frequency $K_{1} / \gamma_{1} d^{2}$ so that $\phi$, and consequently $C$ and $\phi^{\prime}$, are almost independent of time. In this limit, one can average over time the previous equation and replace $D_{z}$ by its rms value which is equivalent to replace $V_{a}$ by its rms value $V$.

To continue, we must now distinguish the two types of samples.

\section{- Antiparallel samples}

In these samples $\phi(0)=\phi(d)=\phi_{a}$ and the calculation is simple because the solution is symmetrical with respect to the mid-plane: $\phi(d-z)=\phi(z)$. As a consequence the maximum tilt angle $\phi_{m}$ is reached at $z=d / 2$ and the equations can be integrated over the half-thickness. In this case, the 
calculation shows after making the change of variable $z \rightarrow \phi$ in Eq. (A4) and by using the first integral (A7) that the capacitance is given by the following parametric equations:

$$
\frac{V}{V_{c}}=\frac{2}{\pi} \int_{\phi_{a}}^{\phi_{m}} \mathrm{~d} \phi \sqrt{\frac{\left(1+\gamma \sin ^{2} \phi_{m}\right)\left(1+\kappa \sin ^{2} \phi\right)}{\left(1+\gamma \sin ^{2} \phi\right)\left(\sin ^{2} \phi_{m}-\sin ^{2} \phi\right)}}
$$

and

$$
\frac{C}{C_{\perp}}=\frac{2}{\pi} \frac{V_{c}}{V} \int_{\phi_{a}}^{\phi_{m}} \mathrm{~d} \phi \sqrt{\frac{\left(1+\gamma \sin ^{2} \phi_{m}\right)\left(1+\gamma \sin ^{2} \phi\right)\left(1+\kappa \sin ^{2} \phi\right)}{\sin ^{2} \phi_{m}-\sin ^{2} \phi}}
$$

where $V_{c}=\pi \sqrt{\frac{K_{1}}{\epsilon_{0} \epsilon_{a}}}$ is the Fredericksz critical voltage. These equations are similar to Eqs. (7) and (8) given in the main text after making the change of variable $\sin \phi=\sin \phi_{m} \sin \psi$. Note that these equations apply at all voltages when $\phi_{a} \neq 0$ since the Freedericsz threshold disappears in this case.

From these formulas it is also possible to find the asymptotic behavior of the capacitance at large voltage. The calculation is the same as in Ref. [27] and yields

$$
\frac{C}{C_{\perp}} \rightarrow 1+\gamma-\frac{2 \gamma}{\pi} \sqrt{1+\gamma} \frac{V_{c}}{V} \int_{\sin \phi_{a}}^{1} \sqrt{\frac{1+\kappa x^{2}}{1+\gamma x^{2}}} \mathrm{~d} x \quad \text { when } \quad V \gg V_{c}
$$

This formula shows that $C / C_{\perp} \rightarrow 1+\gamma$, independently of the value of $\phi_{a}$, when $1 / V \rightarrow 0$, as in a perfect planar sample. By contrast, the slope of the curve $C(1 / V)$ depends on $\phi_{a}$. This result shows that the pretilt angle must be taken into account for measuring $\kappa$ from the slope of this curve, a point that was not mentioned in Ref. [27].

\section{- Parallel samples}

In these samples $\phi(0)=-\phi_{a}$ and $\phi(d)=\phi_{a}$. Because of these boundary conditions one has $\phi(d / 2)=0$ at low voltages, as in a perfect sample, which was not the case in antiparallel samples in which $\phi(d / 2) \geq \phi_{a}$ whatever the applied voltage. For this reason there still exists a critical voltage $V_{c}^{\prime}$ slightly different from $V_{c}$ below which an antisymmetric solution develops with $\phi(d-z)=-\phi(z)$ and $\phi(d / 2)=0$ and above which a asymmetric solution develops with the maximum tilt angle $\phi_{m}$ at a position $z_{m} \neq d / 2$.

A straightforward calculation shows that below the onset of instability the capacitance is given by the following parametric equations:

$$
\frac{V}{V_{c}}=\frac{2}{\pi} \int_{0}^{\phi_{a}} \mathrm{~d} \phi \sqrt{\frac{\left(1+\kappa \sin ^{2} \phi\right)\left(1+\gamma \sin ^{2} \phi_{a}\right) \eta}{\left(1+\gamma \sin ^{2} \phi\right)\left[\sin ^{2} \phi_{a}\left(1+\gamma \sin ^{2} \phi\right)-\eta \sin ^{2} \phi\left(1+\gamma \sin ^{2} \phi_{a}\right)\right]}} \quad \text { for } \quad V<V_{c}^{\prime}
$$

and

$$
\frac{C}{C_{\perp}}=\frac{2}{\pi} \frac{V_{c}}{V} \int_{0}^{\phi_{a}} \mathrm{~d} \phi \sqrt{\frac{\left(1+\kappa \sin ^{2} \phi\right)\left(1+\gamma \sin ^{2} \phi\right)\left(1+\gamma \sin ^{2} \phi_{a}\right) \eta}{\sin ^{2} \phi_{a}\left(1+\gamma \sin ^{2} \phi\right)-\eta \sin ^{2} \phi\left(1+\gamma \sin ^{2} \phi_{a}\right)}} \quad \text { for } \quad V<V_{c}^{\prime}
$$

by taking for parameter $\eta=\frac{\pi^{2}}{\phi_{m}^{\prime 2} d^{2}} \frac{\sin ^{2} \phi_{a}}{1+\gamma \sin ^{2} \phi_{a}} \frac{C^{2}}{C_{\perp}^{2}} \frac{V^{2}}{V_{c}^{2}}$ with $\phi_{m}^{\prime}=\phi^{\prime}(d / 2)$. From these formulas, Eq. (10) can be easily found by noticing that $\eta \rightarrow 0$ when $V \rightarrow 0$.

Above the onset of instability, the solution is asymmetric and passes through a maximum $\phi_{m}$ at a position $z_{m} \neq d / 2$. As $z_{m}$ is unknown, the equations must be integrated over the whole sample 

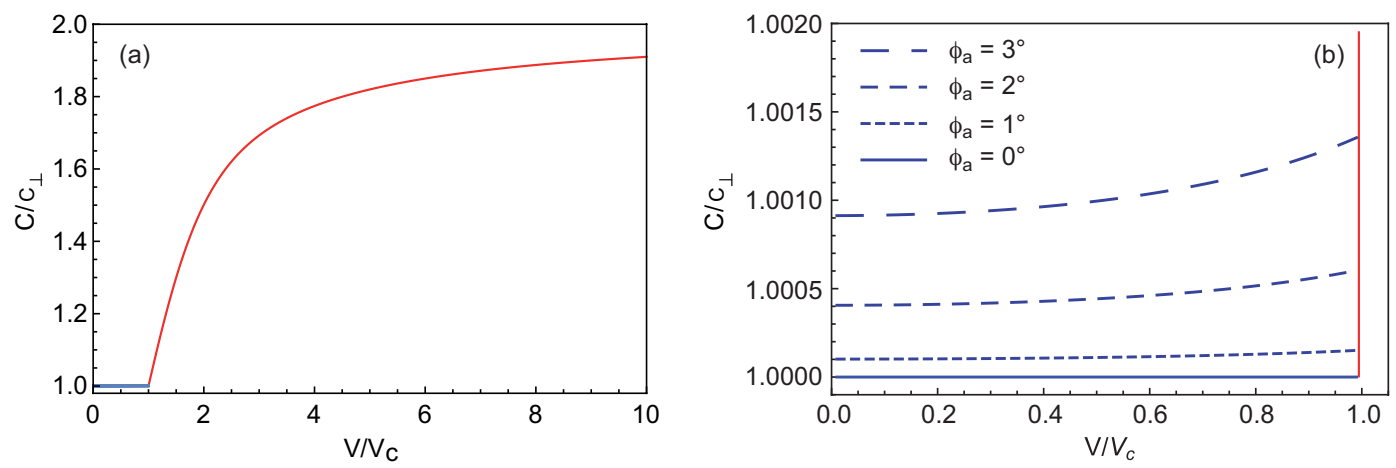

Figure A1. Typical capacitance curves calculated in a parallel sample by taking $\gamma=\kappa=1$ and $\phi_{a}=0,1,2,3^{\circ}$. The curves are identical above the onset of instability (a). On the other hand, they slightly differ below the onset of instability as shown in (b).

thickness which gives

$$
\begin{aligned}
\frac{V}{V_{c}}= & \frac{1}{\pi}\left\{\int_{-\phi_{a}}^{\phi_{m}} \mathrm{~d} \phi \sqrt{\frac{\left(1+\gamma \sin ^{2} \phi_{m}\right)\left(1+\kappa \sin ^{2} \phi\right)}{\left(1+\gamma \sin ^{2} \phi\right)\left(\sin ^{2} \phi_{m}-\sin ^{2} \phi\right)}}\right. \\
& \left.+\int_{\phi_{a}}^{\phi_{m}} \mathrm{~d} \phi \sqrt{\frac{\left(1+\gamma \sin ^{2} \phi_{m}\right)\left(1+\kappa \sin ^{2} \phi\right)}{\left(1+\gamma \sin ^{2} \phi\right)\left(\sin ^{2} \phi_{m}-\sin ^{2} \phi\right)}}\right\} \text { for } V>V_{c}^{\prime}
\end{aligned}
$$

and

$$
\begin{aligned}
\frac{C}{C_{\perp}}= & \frac{2}{\pi} \frac{V}{V_{c}}\left\{\int_{-\phi_{a}}^{\phi_{m}} \mathrm{~d} \phi \sqrt{\frac{\left(1+\gamma \sin ^{2} \phi_{m}\right)\left(1+\gamma \sin ^{2} \phi\right)\left(1+\kappa \sin ^{2} \phi\right)}{\sin ^{2} \phi_{m}-\sin ^{2} \phi}}\right. \\
& \left.+\int_{\phi_{a}}^{\phi_{m}} \mathrm{~d} \phi \sqrt{\frac{\left(1+\gamma \sin ^{2} \phi_{m}\right)\left(1+\gamma \sin ^{2} \phi\right)\left(1+\kappa \sin ^{2} \phi\right)}{\sin ^{2} \phi_{m}-\sin ^{2} \phi}}\right\} \text { for } V>V_{c}^{\prime}
\end{aligned}
$$

If $h(\phi)=h(-\phi)$ then $\int_{-\phi_{a}}^{0} h(\phi) \mathrm{d} \phi=\int_{0}^{\phi_{a}} h(\phi) \mathrm{d} \phi$ and $\int_{-\phi_{a}}^{\phi_{m}} h(\phi) \mathrm{d} \phi+\int_{\phi_{a}}^{\phi_{m}} h(\phi) \mathrm{d} \phi=2 \int_{0}^{\phi_{m}} \mathrm{~d} \phi$. By using this identity in the two previous equations, one obtains

$$
\frac{V}{V_{c}}=\frac{2}{\pi} \int_{0}^{\phi_{m}} \mathrm{~d} \phi \sqrt{\frac{\left(1+\gamma \sin ^{2} \phi_{m}\right)\left(1+\kappa \sin ^{2} \phi\right)}{\left(1+\gamma \sin ^{2} \phi\right)\left(\sin ^{2} \phi_{m}-\sin ^{2} \phi\right)}} \text { for } \quad V>V_{c}^{\prime}
$$

and

$$
\frac{C}{C_{\perp}}=\frac{2}{\pi} \frac{V}{V_{c}} \int_{0}^{\phi_{m}} \mathrm{~d} \phi \sqrt{\frac{\left(1+\gamma \sin ^{2} \phi_{m}\right)\left(1+\gamma \sin ^{2} \phi\right)\left(1+\kappa \sin ^{2} \phi\right)}{\sin ^{2} \phi_{m}-\sin ^{2} \phi}} \text { for } \quad V>V_{c}^{\prime}
$$

where $\eta=\sin ^{2} \phi_{m}$. A remarkable result is that, above the onset of instability, the capacitance is given by the same parametric equations as in a perfect planar sample. This solution disappears when $z_{m}=d$ and $\theta_{m}=\theta_{a}$. This condition fixes the onset of instability $V_{c}^{\prime}$. By using Eq. (A15) one obtains $V_{c}^{\prime}=\alpha V_{c}$ with

$$
\alpha=\frac{2}{\pi} \int_{0}^{\phi_{a}} \mathrm{~d} \phi \sqrt{\frac{\left(1+\gamma \sin ^{2} \phi_{a}\right)\left(1+\kappa \sin ^{2} \phi\right)}{\left(1+\gamma \sin ^{2} \phi\right)\left(\sin ^{2} \phi_{a}-\sin ^{2} \phi\right)}}
$$

Typical capacitance curves calculated by taking $\gamma=\kappa=1$ for different values of $\phi_{a}$ are shown in Fig. A1. They superpose above the onset of instability and shift from 1 by less than $510^{-4}$ below the 

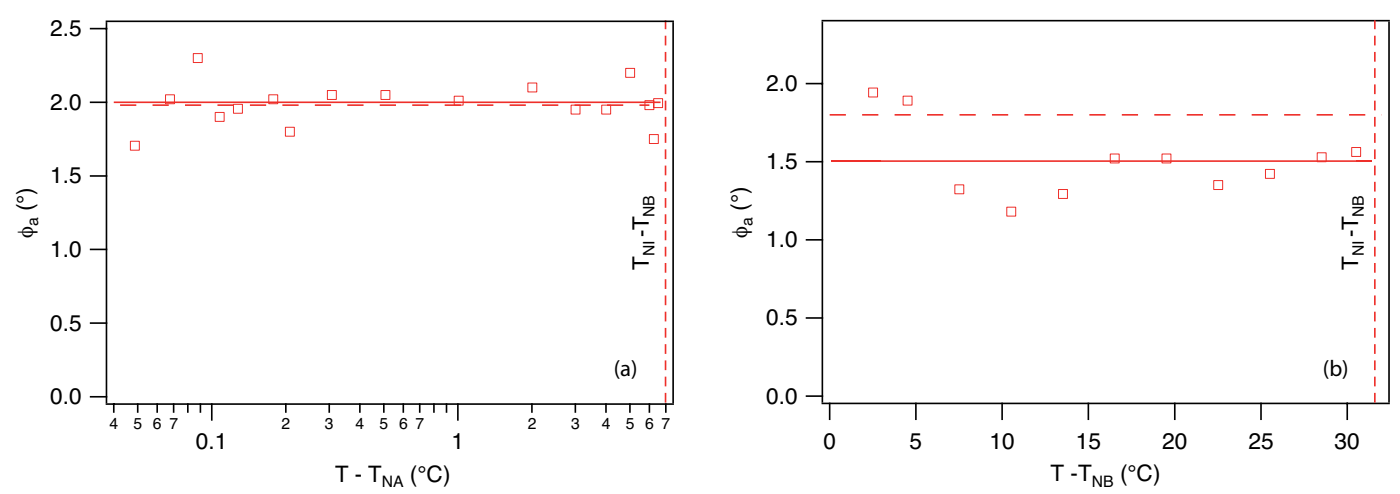

Figure A2. Pretilt angle measured with the capacitive method at large voltage in $8 \mathrm{CB}$ (a) and in the mixture $8 \mathrm{CB} / \mathrm{CB} 7 \mathrm{CB}$ (b). In $8 \mathrm{CB}$, the average value found by this method $\left(\phi_{a} \approx 2^{\circ}\right.$, solid line) is the same as the value found previously by using the optical method or the capacitive method at small voltages (dashed line). By contrast, the value of $\phi_{a}$ found by this method in the mixture $8 \mathrm{CB} / \mathrm{CB} 7 \mathrm{CB}\left(\phi_{a} \approx 1.5^{\circ} \mathrm{C}\right.$, solid line $)$ is a bit smaller than the value $\left(\phi_{a} \approx 1.8^{\circ}\right.$, dashed line) found by using the optical method or the capacitive method at small voltages. This shift will be explained in Appendix C.

onset when $\phi_{a}=2^{\circ}$. This calculation shows that the parallel samples behave almost like a perfect planar sample as long as $\phi_{a}$ remains small.

For completeness, we also calculated the solution for a parallel sample in which a $\pi$-wall has formed.

- Parallel samples with a $\pi$-wall

In that case, one has $\phi(0)=\phi_{a}$ and $\phi(d)=\pi-\phi_{a}$ and the parametric equations become

$$
\frac{V}{V_{c}}=\frac{2}{\pi} \int_{\phi_{a}}^{\pi / 2} \mathrm{~d} \phi \sqrt{\frac{(1+\gamma)\left(1+\kappa \sin ^{2} \phi\right)}{\left(1+\gamma \sin ^{2} \phi\right)\left(1-\eta \sin ^{2} \phi\right)}}
$$

and

$$
\frac{C}{C_{\perp}}=\frac{2}{\pi} \frac{V_{c}}{V} \int_{\phi_{a}}^{\pi / 2} \mathrm{~d} \phi \sqrt{\frac{(1+\gamma)\left(1+\gamma \sin ^{2} \phi\right)\left(1+\kappa \sin ^{2} \phi\right)}{1-\eta \sin ^{2} \phi}}
$$

where the parameter is defined to be $\eta=1-\frac{\gamma(1+\gamma)(1+\kappa) d^{2} \phi_{m}^{\prime 2}}{\pi^{2}} \frac{C_{\perp}^{2}}{C^{2}} \frac{V_{c}^{2}}{V^{2}}$ with $\phi_{m}^{\prime}=\phi^{\prime}(d / 2)$.

From these formulas it is still possible to find the asymptotic behavior of the capacitance at large voltage. This calculation gives

$$
\frac{C}{C_{\perp}} \rightarrow 1+\gamma-\frac{2 \gamma}{\pi} \sqrt{1+\gamma} \int_{\sin \phi_{a}}^{1} \sqrt{\frac{1+\kappa x^{2}}{1+\gamma x^{2}}} \mathrm{~d} x \frac{V_{c}}{V} \quad \text { when } \quad V \gg V_{c}
$$

This formula is exactly the same as in an antiparallel sample and shows that $C / C_{\perp} \rightarrow 1+\gamma$ when $1 / V \rightarrow 0$.

By noticing that $\int_{\sin \phi_{a}}^{1} \sqrt{\frac{1+\kappa x^{2}}{1+\gamma x^{2}}} \mathrm{~d} x \approx \int_{0}^{1} \sqrt{\frac{1+\kappa x^{2}}{1+\gamma x^{2}}}-\phi_{a}=I-\phi_{a}$, one also see from Eq. (A10) and (A20) that measuring the slope $p$ of the curves $C(1 / V)$ at large voltages in antiparallel samples (or parallel samples in which a $\pi$-wall has formed) should allow to measure the pretilt angle by using the formula

$$
\phi_{a}=I-\frac{\pi p}{2 V_{c} \gamma \sqrt{1+\gamma}}
$$

Note here that this formula is applicable only for strong anchoring energy. To test this method, we measured the slope $p$ in $8 \mathrm{CB}$ and the mixture $8 \mathrm{CB} / \mathrm{CB} 7 \mathrm{CB}$ and we calculated $\phi_{a}$ by using this formula 
and the values of $\kappa$ and $\gamma$ measured previously. In this way we found that $\phi_{a}$ was approximately constant, of the order of $2^{\circ}$ in $8 \mathrm{CB}$ and $1.5^{\circ}$ in the mixture $8 \mathrm{CB} / \mathrm{CB} 7 \mathrm{CB}$ (Fig. A2). For $8 \mathrm{CB}$, we note that this value is the same as that given before to within experimental errors. By contrast, the value found in the mixture $8 \mathrm{CB} / \mathrm{CB} 7 \mathrm{CB}$ is slightly smaller than that given previously (of the order of $1.8^{\circ} \mathrm{C}$ ). This disagreement is not accidental but is due to the finite value of the anchoring energy, smaller in the mixture $8 \mathrm{CB} / \mathrm{CB} 7 \mathrm{CB}$ than in pure $8 \mathrm{CB}$. This point will be discussed in Appendix $\mathrm{C}$. Before that, we analyze the role of flexoelectricity.

\section{Appendix B. Role of the flexoelectric effect}

So far we have neglected flexoelectricity and found a good agreement between optical and capacitive measurements. This result is a bit surprising. Indeed, in a recent paper, Brown and Mottram have shown that the value of $K_{3}$ found by using the capacitive method is overestimated when the flexoelectricity is neglected in the calculation [7]. As emphasized by the authors of Ref. [5], this effect could be dominant in liquid crystals as the mixture $8 \mathrm{CB} / \mathrm{CB} 7 \mathrm{CB}$ in which $K_{3}$ is very small and where significant flexoelectric effects could be present, as already observed in pure CB7CB [38, 39]. Flexoelectric effects could also bias the measurement of $K_{3}$ in the optical method, but in a different manner. For this reason, we expected to find values of $K_{3}$ slightly different by using the two methods, but it was not the case. In this appendix, we explain why the two methods are in fact insensible to flexoelectricity in our experiments. To start, we analyze the role of flexoelectricity in the optical method.

\section{B.0.1 In the optical measurements}

We first consider the case of an insulating nematic. Our notations are the same as before. Because the director field is distorted there exists a net flexoelectric polarization $\vec{P}=e_{1} \vec{n} \nabla \cdot \vec{n}-e_{3} \vec{n} \times \vec{\nabla} \times \vec{n}$ of $z$-component

$$
P_{z}=e^{\star} \sin \phi \cos \phi \phi^{\prime}
$$

where $e^{\star}=e_{1}+e_{3}$ is the bulk flexoelectric coefficient. Because $\vec{\nabla} \cdot \vec{P} \neq 0$ inside the sample, there are polarization charges and the electric field $E$ is no longer equal to zero. For this reason the total free energy contains electric terms and reads by setting $E=-\frac{\partial V}{\partial z}$ :

$$
F=\int_{0}^{d} \mathrm{~d} z\left\{\frac{1}{2} K(\phi) \phi^{\prime 2}-\frac{1}{2} \epsilon_{0} \epsilon(\phi) V^{\prime 2}+e^{\star} \sin \theta \cos \theta V^{\prime} \phi^{\prime}\right\}
$$

Minimizing with respect to $\phi$ and $V$ gives the torque equation

$$
\left(1+\kappa \sin ^{2} \phi\right) \phi^{\prime \prime}+\frac{1}{2} \kappa \sin 2 \phi \phi^{2}+\frac{e^{\star}}{2 K_{1}} \sin 2 \phi V^{\prime \prime}+\frac{\epsilon_{0} \epsilon_{a}}{2 K_{1}} \sin 2 \phi V^{\prime 2}=0
$$

and the Maxwell equation $\vec{\nabla} \cdot \vec{D}=0$ which reads

$$
\left(1+\gamma \sin ^{2} \phi\right) V^{\prime \prime}+\gamma \sin 2 \phi V^{\prime} \phi^{\prime}-\frac{e^{\star}}{\epsilon_{0} \epsilon_{\perp}}\left[\frac{\sin 2 \phi}{2} V^{\prime \prime}+\cos 2 \phi \phi^{\prime 2}\right]=0
$$



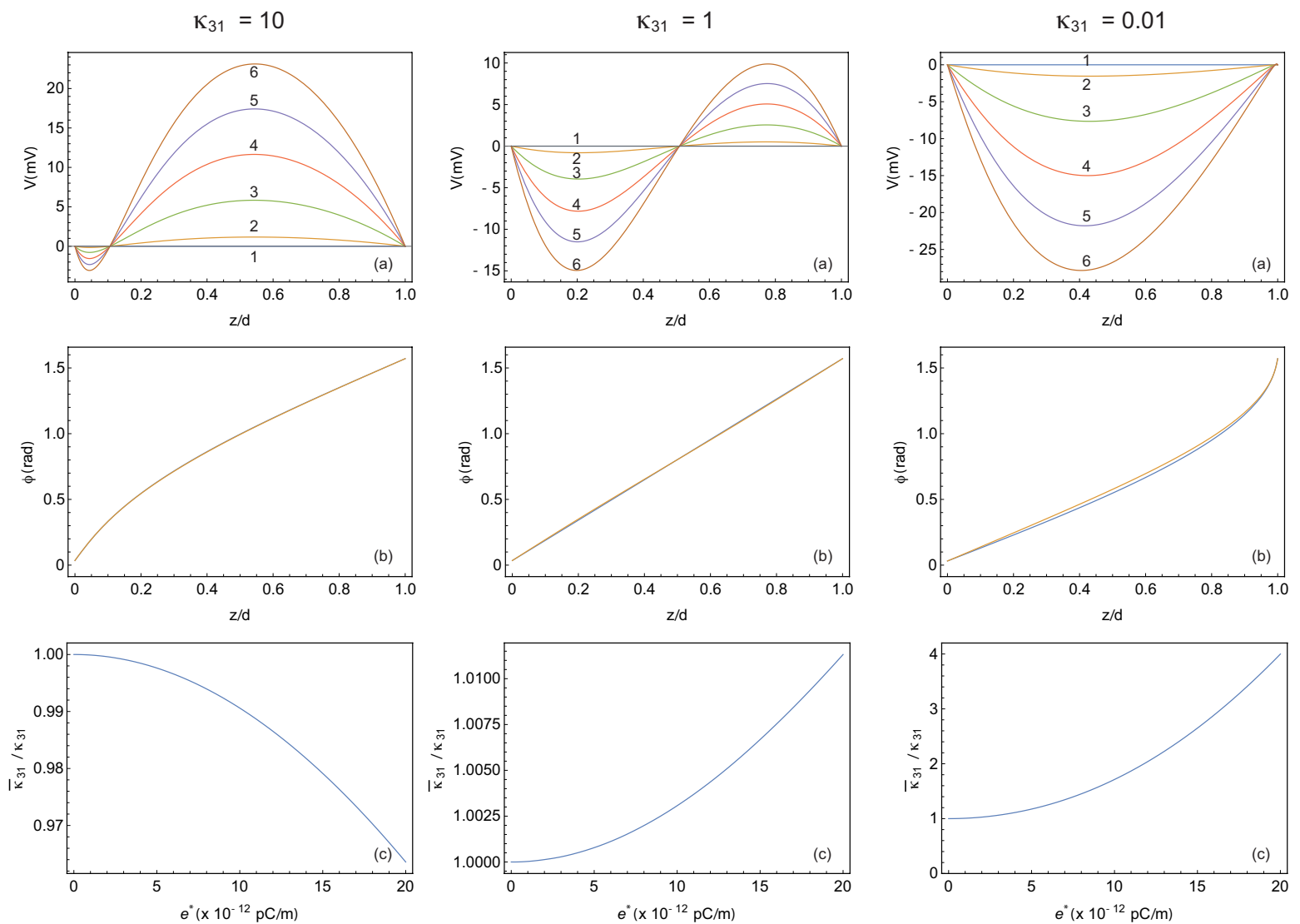

Figure B1. Voltage and angle profiles $(\mathrm{a}, \mathrm{b})$ calculated for different values of the flexoelectric coefficient. In graphs (a), $e^{\star}(p C / m)=0$ (curve 1), 1 (curve 2), 5 (curve 3), 10 (curve 4), 15 (curve 5 ) and 20 (curve 6). In graphs (b) only the curves calculated at $e^{\star}=0$ (bottom curve) and $e^{\star}=20 \mathrm{pN} / \mathrm{m}$ (top curve) are shown. When $\kappa_{31}=10$ or 1 these curves are almost superimposed. Graphs (c) show that the value of $\kappa_{31}$ measured by neglecting flexoelectricity (denoted by $\bar{\kappa}_{31}$ ) can strongly differ from its bare value $\kappa_{31}$ when $\kappa_{31}$ is very small and $e^{\star}>5 \mathrm{pN} / \mathrm{m}$. These graphs have been calculated in the limit of an insulating nematic sample.

Under strong anchoring conditions, these two equations are subjected to the boundary conditions

$$
\left\{\begin{array}{r}
\phi(0)=\phi_{a} \\
\phi(d)=\frac{\pi}{2} \\
V(0)=V(d)=0
\end{array}\right.
$$

knowing that in our experiments, the samples are prepared between two ITO electrodes that are short-circuited by contact with the metallic wires used as a spacer. These equations can be solved numerically with Mathematica. From the solution, the retardation $\delta$ can then be calculated by using Eq. (4). Finally, one can calculate by using Eqs. (2) and (4) the value of $\kappa_{31}$ that would give the same retardation $\delta$ in the absence of flexoelectricity. This value, denoted by $\bar{\kappa}_{31}$, is the value that is effectively measured when the flexoelectricity is neglected. We calculated $\bar{\kappa}_{31}$ in three cases: when $\kappa_{31}$ is large (case of $8 \mathrm{CB}$ close to $T_{N A}$ ), when $\kappa_{31}$ is close to 1 (as in usual nematics) and when $\kappa_{31}$ is very small (case of the mixture $8 \mathrm{CB} / \mathrm{CB} 7 \mathrm{CB}$ close to $T_{N B}$ ). For the calculations we took $K_{1}=10 \mathrm{pN}$, $\epsilon_{\perp}=6, \epsilon_{\|}=12, \phi_{a}=2^{\circ}, n_{o}=1.55$ and $\Delta n=0.15$. The results are reported in Fig. B1. In each case, three graphs are shown. The first two $(\mathrm{a}, \mathrm{b})$ show the voltage and angle profiles calculated for typical values of $e^{\star}$ [40] and the third one (c) shows the evolution of $\bar{\kappa}_{31} / \kappa_{31}$ as a function of $e^{\star}$. The latter graphs show that the error made on $\kappa_{31}$ when neglecting the flexoelectricity is very small when $\kappa_{31}$ is 

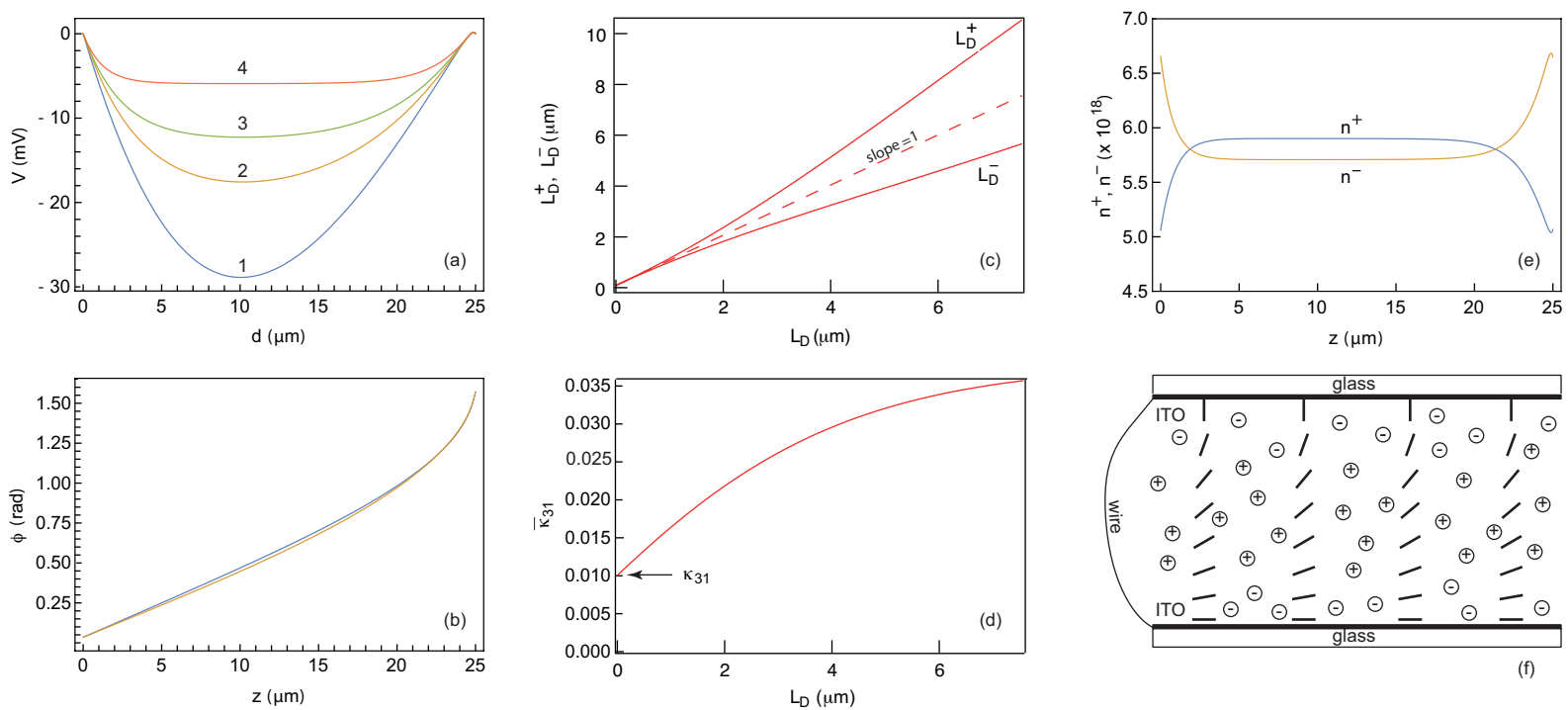

Figure B2. (a) Potential profiles for different values of the Debye length. From (1) to (4) $L_{D}=\infty, 4.24,2.83,1.41 \mu \mathrm{m}$; (b) Angle profile for $L_{D}=\infty$ (top curve) and $L_{D}=1.41 \mu \mathrm{m}$ (bottom curve); (c) The two lengths $L_{D}^{+}$and $L_{D}^{-}$as a function of the Debye length; (d) Value $\bar{\kappa}_{31}$ of $\kappa_{31}$ measured by neglecting the flexoelectricity as a function of the Debye length. This graph shows that $\bar{\kappa}_{31} \rightarrow \kappa_{31}$ when $L_{D} \rightarrow 0$; (e) Distributions of the ions calculated when $L_{D}=976 \mathrm{~nm}$; (f) Schematic representation of the director field and the distribution of the ions inside the sample. The wire on the left indicates that the two ITO layers are electrically connected, which justifies the boundary condition on the potential given in Eq. (3).

large or of order unity (case of $8 \mathrm{CB}$ ). One will note that the $\kappa_{31}$ is generally overestimed except when it is very large. By contrast, the error becomes very important when $\kappa_{31}$ is small and $e^{\star}>5 \mathrm{pC} / \mathrm{m}$ as shown in graph (c) of the column $\kappa_{31}=0.01$. This could be the case in our mixture $8 \mathrm{CB} / \mathrm{CB} 7 \mathrm{CB}$, knowing that in pure CB7CB significant flexoelectric effects have been reported [38, 39].

This result must, however, be qualified given that our samples are not pure and contain free ions that can screen the electric field created by the flexoelectric charges. This is clearly the case in our samples of $8 \mathrm{CB} / \mathrm{CB} 7 \mathrm{CB}$ in which we measured charge relaxation frequencies of the order of $1.5 \mathrm{kHz}$. To show that ions can screen the flexoelectric charges, we have reconsidered this problem by taking into account the ions. For simplicity, we assumed that our sample contains $\bar{n}$ ions of charge $+e$ and $\bar{n}$ ions of charge $-e$ per unit volume. We also assumed that the surfaces are not charged. In the presence of flexoelectricity, the ions move and the local concentration of anions $n^{-}$becomes different of the local concentration of cations $n_{+}$leading to a net free charge $\rho=\left(n_{+}-n_{-}\right) e \neq 0$. In this condition the free energy to minimize reads

$$
F=\int_{0}^{d} \mathrm{~d} z\left\{\frac{1}{2} K(\phi) \phi^{\prime 2}-\frac{1}{2} \epsilon_{0} \epsilon(\phi) V^{\prime 2}+e^{\star} \sin \theta \cos \theta V^{\prime} \phi^{\prime}+\rho V\right\}
$$

Minimizing with respect to $\phi$ gives back the torque equation (B4) that remains unchanged whereas the Maxwell equation obtained by minimizing with respect to $V$ becomes $\nabla \cdot \vec{D}=\rho$, that is

$$
\frac{\mathrm{d}}{\mathrm{d} z}\left[\epsilon(\phi) V^{\prime}-\frac{e^{\star}}{\epsilon_{0}} \sin \phi \cos \phi \phi^{\prime}\right]=\rho
$$

At equilibrium, we assume that the concentrations of cations and anions follow Boltzmann statistics so that

$$
n^{+}=n_{0}^{+} e^{-\frac{V}{V^{\star}}} \quad \text { and } \quad n^{-}=n_{0}^{-} e^{\frac{V}{V^{\star}}}
$$

where $V^{\star}=k_{B} T / e$ is the thermal potential of the order of $25 \mathrm{mV}$ at room temperature, and $n_{0}^{+}$ and $n_{0}^{-}$are the local concentrations of cations and anions at the place where $V=0$. Under this 
assumption, the conduction current exactly equilibrates the diffusion current for both kinds of ions, so that there is no net current inside the sample as required at equilibrium. By introducing the lengths

$$
L_{D}^{+}=\sqrt{\frac{\epsilon_{0} \bar{\epsilon} k_{B} T}{2 n_{0}^{+} e^{2}}} \text { and } L_{D}^{-}=\sqrt{\frac{\epsilon_{0} \bar{\epsilon} k_{B} T}{2 n_{0}^{-} e^{2}}}
$$

where $\bar{\epsilon}=\frac{\epsilon_{\|}+2 \epsilon_{\perp}}{3}$, the Maxwell equation $\vec{\nabla} \cdot \vec{D}=\rho$ writes in the form of the Poisson-Boltzmann equation

$$
\frac{\mathrm{d}}{\mathrm{d} z}\left[\frac{\epsilon(\phi)}{\bar{\epsilon}} V^{\prime}-\frac{e^{\star}}{\bar{\epsilon} \epsilon_{0}} \sin \phi \cos \phi \phi^{\prime}\right]=\frac{1}{2} V^{\star}\left(\frac{1}{L_{D}^{-2}} e^{\frac{V}{V \star}}-\frac{1}{L_{D}^{+2}} e^{-\frac{V}{V \star}}\right)
$$

Note that since the sample has a finite thickness, the two lengths $L_{D}^{+}$and $L_{D}^{-}$are different from the Debye length defined to be $L_{D}=\sqrt{\frac{\epsilon_{0} \bar{\epsilon} k_{B} T}{2 \bar{n} e^{2}}}$. For this reason one more equation is necessary to calculate $L_{D}^{+}$and $L_{D}^{-}$. This equation comes from the global conservation of the charges and reads

$$
\bar{n}=\frac{1}{d} \int_{0}^{d} n_{0}^{+} e^{-\frac{V}{V^{\star}}} \mathrm{d} z=\frac{1}{d} \int_{0}^{d} n_{0}^{-} e^{\frac{V}{V^{\star}}} \mathrm{d} z
$$

or, equivalently,

$$
\frac{L_{D}^{-2} \int_{0}^{d} e^{-\frac{V}{V^{\star}}} \mathrm{d} z}{L_{D}^{+2} \int_{0}^{d} e^{\frac{V}{V^{\star}}} \mathrm{d} z}=1
$$

To see how the ions screen out the flexoelectric charges, we have solved numerically with Mathematica the equations (B4), (B10) and (B12) in the case $\kappa_{31}=0.01$ for decreasing value of the Debye length $L_{D}$ by taking $e^{\star}=20 \mathrm{pC} / \mathrm{m}$ (which is large) and the same values as before for the other parameters. The equations were solved by successive approximations by varying slowly the Debye length and by using a shooting method. Typical curves are shown in Fig. B2. Graph (a) shows that $V \rightarrow 0$ when the $L_{D} \rightarrow 0$ because of the screening by the ions. As a consequence, the angle profile changes slightly (Graph (b)) and the value $\bar{\kappa}_{31}$ measured by neglecting flexoelectricity tends to $\kappa_{31}$ when $L_{D} \rightarrow 0$, as shown in graph (d). Graph (c) shows that $L_{D}^{+}=L_{D}^{-}=L_{D}$ when $L_{D} \rightarrow 0$. Finally, graph (e), calculated for $L_{D}=345 \mathrm{~nm}$, and the drawing in (f) show the distribution of the ions inside the cell.

In practice, our samples are impure and contain ions. In the case of $8 \mathrm{CB}$, we measured $f_{c} \approx 200 \mathrm{~Hz}$ and in the mixture $8 \mathrm{CB} / \mathrm{CB} 7 \mathrm{CB}, f_{c}$ was larger, of the order of $1.5 \mathrm{kHz}$. According to Bazant et al. $[41]$

$$
L_{D} \approx \sqrt{\frac{D}{2 \pi f_{c}}}
$$

where $D$ is an average value of the diffusion coefficients of ions in the LC. By taking for typical values $D=10 \mu \mathrm{m}^{2} / \mathrm{s}$ [42], one calculates $L_{D} \sim 90 \mathrm{~nm}$ in $8 \mathrm{CB}$ and $L_{D} \sim 30 \mathrm{~nm}$ in the mixture $8 \mathrm{CB} / \mathrm{CB} 7 \mathrm{CB}$. The immediate consequence is that flexoelectric effects are completely screened in our experiments as we can see from Fig. (B2)(d). For this reason, we believe that the optical method gives the good value of $\kappa_{31}$ in our experiments. 


\section{B.0.2 In the capacitance measurements}

As before, we start with the case of an insulating nematic. In the absence of free charges, $\vec{\nabla} \times \vec{D}=0$ and $D_{z}=\epsilon_{0} \epsilon(\phi) E+P_{z}$ where $P_{z}$ is given in Eq. (B1), only depends on time. As a consequence the free energy to minimize becomes

$$
F=\int_{0}^{d} \mathrm{~d} z\left\{\frac{1}{2} K(\phi) \phi^{\prime 2}-\frac{1}{2} D_{z} E-\frac{1}{2} P_{z} E\right\}
$$

As $V_{a}=\int_{0}^{d} E \mathrm{~d} z$, and $D_{z}$ is a function of $t$ only, we obtain

$$
F=-\frac{1}{2} D_{z} V_{a}+\frac{1}{2} \int_{0}^{d} \mathrm{~d} z\left\{K(\phi) \phi^{\prime 2}-P_{z} E\right\}
$$

To calculate $D_{z}$ we write that $V_{a}=\int_{0}^{d} E \mathrm{~d} z$ with $E=\left(D_{z}-P_{z}\right) / \epsilon_{0} \epsilon(\phi)$. This gives after integration

$$
V_{a}=\frac{D_{z}}{\epsilon_{0}} \int_{0}^{d} \frac{\mathrm{d} z}{\epsilon(\phi)}-\frac{e^{\star}}{2 \epsilon_{0} \epsilon_{a}} \log \left[\frac{\epsilon[\phi(d)]}{\epsilon[\phi(0)]}\right]
$$

In our experiments $\phi(d)=\phi(0)$ (antiparallel samples) or $\phi(d)=-\phi(0)$ (parallel samples) or $\phi(d)=$ $\pi-\phi(0)$ (parallel sample with a $\pi$-wall). In these conditions $\epsilon[\phi(d)]=\epsilon[\phi(0)]$ and the log term vanishes in the previous equation. As a consequence, Eq. (A4) remains unchanged in our samples in the presence of flexoelectricity

$$
D_{z}=\frac{\epsilon_{0} V_{a}}{\int_{0}^{d} \epsilon(\phi)^{-1} \mathrm{~d} z}
$$

Note that this conclusion still holds if the anchoring energy is finite, but the same on the two surfaces. In these conditions, the free energy to minimize reads after replacing $D_{z}$ and $P_{z}$ by their expressions given in Eqs. (B17) and (B1) in Eq. (B15)

$$
F=\int_{0}^{d} \frac{1}{2} K(\phi) \phi^{\prime 2} \mathrm{~d} z-\frac{1}{2} \epsilon_{0} \frac{V_{a}^{2}}{\int_{0}^{d} \epsilon(\phi)^{-1} \mathrm{~d} z}+\frac{e^{\star 2}}{2 \epsilon_{0} \epsilon_{\perp}} \int_{0}^{d} \frac{\sin ^{2} \phi \cos ^{2} \phi^{\prime 2}}{1+\gamma \sin ^{2} \phi} \mathrm{d} z
$$

Minimizing this equation with respect to $\phi$ gives after integration [43]

$$
\left[1+\kappa^{\star}(\phi) \sin ^{2} \phi\right] \phi^{\prime 2}=\frac{D_{z}^{2}}{K_{1} \epsilon_{0} \epsilon_{\perp}} \frac{\sin ^{2} \phi_{m}-\sin ^{2} \phi}{\left(1+\gamma \sin ^{2} \phi\right)\left(1+\gamma \sin ^{2} \phi_{m}\right)}
$$

where

$$
\kappa^{\star}(\phi)=\kappa+\frac{e^{\star 2}}{\epsilon_{0} \epsilon_{\perp} K_{1}} \frac{\cos ^{2} \phi}{1+\gamma \sin ^{2} \phi}
$$

This equation is the same as Eq. (A7) on condition to replace $\kappa$ by $\kappa^{\star}$. This calculation shows that, in agreement with the results of Ref. [43], the main effect of flexoelectricity is to renormalize the elastic anisotropy. As a consequence all the formula given before apply on condition to replace $\kappa$ by $\kappa^{\star}(\phi)$. Note that in the formulas in which the variables $\psi$ or $x$ are used, $\kappa$ must be replaced by

$$
\kappa^{\star}(\psi)=\kappa+\frac{e^{\star 2}}{\epsilon_{0} \epsilon_{\perp} K_{1}} \frac{1-\eta \sin ^{2} \psi}{1+\gamma \eta \sin ^{2} \psi}
$$



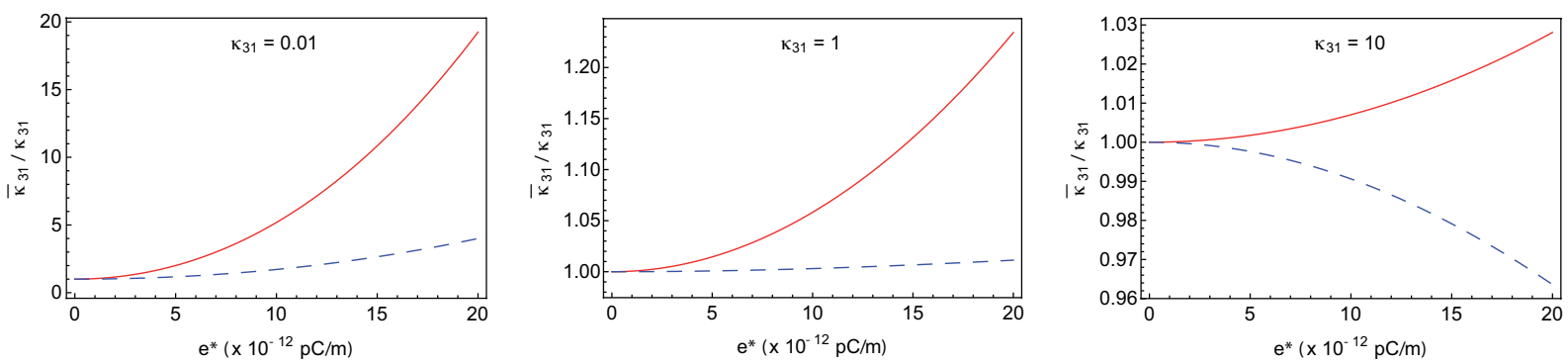

Figure B3. Ratio $\bar{\kappa}_{31} / \kappa_{31}$ measured by the capacitive method (red solid lines) and by the optical method (blue dashed lines) as a function of the flexoelectric coefficient when the sample is isolating. The error made by neglecting flexoelectricity is larger in the capacitive method than in the optical method and increases when $\kappa_{31}$ decreases.

or

$$
\kappa^{\star}(x)=\kappa+\frac{e^{\star 2}}{\epsilon_{0} \epsilon_{\perp} K_{1}} \frac{1-x^{2}}{1+\gamma x^{2}}
$$

We emphasize that, here, the capacitance measured at high frequency changes because $\phi(z)$ changes under the action of the DC flexoelectric component of the electric field $E_{\mathrm{DC}}=-P_{z} / \epsilon_{0} \epsilon(z)$ that adds to the usual AC component $E_{\mathrm{AC}}=D_{z} / \epsilon_{0} \epsilon(z)$.

In order to test the influence of flexoelectricity in the measurements of $\kappa_{31}$, we calculated the value $\bar{\kappa}_{31}$ of $\kappa_{31}$ that would give a fit of the capacitance curve by neglecting flexoelectricity. Calculations were done for antiparallel samples with the same values as before, that is $\phi_{a}=2^{\circ}, K_{1}=10 \mathrm{pN}$ and $\epsilon_{\|}=12$ and $\epsilon_{\perp}=6$ and $\kappa_{31}=0.01,0.1,10$ and $e^{\star}$ varying between 0 and $20 \mathrm{pC} / \mathrm{m}$. The results are reported in Fig. B3 and show that the error made by neglecting flexoelectricity is larger in the capacitive method than in the optical method. It nevertheless remains negligible when $\kappa_{31}$ is large but may become considerable when $\kappa_{31}$ is very small.

As before, this result must be qualified because our samples are not pure and contain ions. For this reason, the static contribution to the electric field due to the flexoelectric effect must be screened out in the capacitive method, in the same manner as in the optical method, even if the measurements are performed in the dielectric regime at frequency $f \gg f_{c}$. This conclusion was confirmed experimentally by fitting the experimental curves with the flexoelectric correction. In all cases, we found that the best fit was obtained by taking $e^{\star}=0$ meaning that in our experiments the flexoelectric effects are absent or screened out by the ions $[44,45]$.

\section{Appendix C. Role of the anchoring energy}

In the previous calculations, we assumed that the anchoring energy $W(\phi)$ was infinite on the plates treated for planar anchoring. In this limit, $\phi_{a}$ is constant. In practice, this energy is always finite and may be supposed of the form given by Rapini and Papoular [46] when angle $\phi$ is close to $\phi_{a}$, that is

$$
W(\phi)=\frac{1}{2} W_{a} \sin ^{2}\left(\phi-\phi_{a}\right)
$$

In that case, the angle on the plates treated for planar anchoring shifts from $\phi_{a}$, which can change the results. In the following, we analyze the role of the anchoring energy, first in the capacitive method and then in the optical method. For the reasons explained in Appendix B, flexoelectric effects are neglected. 


\section{C.1 Role of finite surface anchoring in the capacitance measurements}

As before we distinguish the two types of samples.

\section{- Antiparallel samples}

In these samples, the angles $\phi(0)$ and $\phi(d)$ measured on the plates change when an electric field is applied, but they remain equal by symmetry if the anchoring energy is the same on the two plates. In that case, all the formulas given previously remain valid on condition to replace $\phi_{a}$ by $\phi(0)$. To calculate $\phi(0)$ we write the surface torque equation on the plate at $z=0$. This gives

$$
\frac{W_{a}}{2} \sin 2\left(\phi_{0}-\phi_{a}\right)=K_{1}\left(1+\kappa \sin ^{2} \phi_{0}\right) \phi_{0}^{\prime}
$$

by setting $\phi(0) \equiv \phi_{0}$ and $\phi_{0}^{\prime} \equiv \phi^{\prime}(0)$. Calculating $\phi_{0}^{\prime}$ from the first integral (A7) gives after substitution in Eq. (C2) [20]

$$
\frac{d}{4 l_{p}} \sin \left(2 \phi_{0}-2 \phi_{a}\right)=\sqrt{\frac{\left(\sin ^{2} \phi_{m}-\sin ^{2} \phi_{0}\right)\left(1+\kappa \sin ^{2} \phi_{0}\right)}{1+\gamma \sin ^{2} \phi_{0}}} \int_{\phi_{0}}^{\phi_{m}} \mathrm{~d} \phi \sqrt{\frac{\left(1+\gamma \sin ^{2} \phi\right)\left(1+\kappa \sin ^{2} \phi\right)}{\sin ^{2} \phi_{m}-\sin ^{2} \phi}}
$$

where $l_{p}=K_{1} / W_{a}$ is the so-called anchoring extrapolation length [1,2].

The capacitance curve is obtained by solving this equation together with Eqs. (A8) and (A9) in which $\phi_{a}$ must be replaced by $\phi_{0}$. We recall that these equations apply whatever the value of the applied voltage $V$ when $\phi_{a} \neq 0$ since there is no Freedericskz threshold.

The situation is different in the limiting case $\phi_{a}=0$. The calculations show that there exists a critical voltage given by $V_{c}^{\prime}=\alpha V_{c}$ where $\alpha$ is solution of the following equation [20, 46]:

$$
\frac{d}{l_{p}}=\pi \alpha \tan \left(\frac{\pi \alpha}{2}\right)
$$

In that case, $\phi=0$ and $C=C_{\perp}$ when $V<V_{c}^{\prime}$. When $V>V_{c}^{\prime}$, the capacitance is given by solving Eqs. (A8) and (A9) in which $\phi_{a}$ must be replaced by $\phi_{0}$ given by Eq. (C3).

An interesting point is to analyze what happens in the large voltage limit (in practice for 5 Vrms $<$ $V<20 \mathrm{Vrms}$ ) when $\phi_{m} \rightarrow \pi / 2$. If the anchoring energy is very large, $\phi_{0}$ remains very small and one obtains by using Eqs. (A9) and (C3)

$$
\phi_{0}-\phi_{a} \approx \frac{l_{p}}{d} \frac{\pi}{\sqrt{1+\gamma}} \frac{V}{V_{c}} \frac{C}{C_{\perp}}
$$

At large voltage, Eq. (A10) remains valid on condition to replace $\phi_{a}$ by $\phi_{0}$. By using this equation and Eq. (C5), one obtains

$$
\frac{C}{C_{\perp}} \rightarrow 1+\gamma-\frac{2 \gamma}{\pi} \sqrt{1+\gamma} \frac{V_{c}}{V}\left(\int_{\phi_{a}}^{1} \sqrt{\frac{1+\kappa x^{2}}{1+\gamma x^{2}}} \mathrm{~d} x-\frac{l_{p}}{d} \frac{\pi}{\sqrt{1+\gamma}} \frac{V}{V_{c}} \frac{C}{C_{\perp}}\right) \quad \text { when } \quad V \gg V_{c}
$$

or equivalently

$$
\frac{C}{C_{\perp}} \rightarrow \frac{1+\gamma}{1-2 \gamma \frac{l_{p}}{d}}-\frac{2}{\pi} \frac{\gamma \sqrt{1+\gamma}}{1-2 \gamma \frac{l_{p}}{d}} \int_{\phi_{a}}^{1} \sqrt{\frac{1+\kappa x^{2}}{1+\gamma x^{2}}} \mathrm{~d} x \frac{V_{c}}{V} \quad \text { when } \quad V \gg V_{c}
$$

This equation shows that $C / C_{\perp}$ is still a linear function of $1 / V$. On the other hand, the extrapolation to 0 of this curve does no longer gives $1+\gamma$ but a value slightly larger, that is $(1+\gamma) /\left(1-2 \gamma l_{p} / d\right)$. 


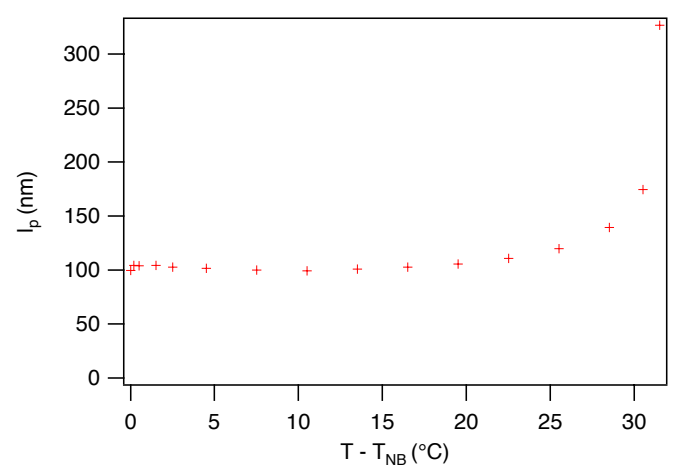

Figure C1. Anchoring extrapolation length $l_{p}$ as a function of temperature measured in the mixture $8 \mathrm{CB} / \mathrm{CB} 7 \mathrm{CB}$.

In a similar way, the slope $p$ of this curve increases since it is divided by the factor $1-2 \gamma l_{p} / d$. This result is important as we shall see later.

\section{- Parallel samples}

In that case the calculations are much more complicated and we did not performed them. In practice there still exists a critical voltage $V_{c}^{\prime}=\alpha V_{c}$ below which the solution remains antisymmetrical and above which it becomes asymmetrical.

The situation simplifies when a $\pi$-wall has formed in the sample. In that case the solution is symmetrical with respect to the mid-plane $z=d / 2$ and $\phi(d)=\pi-\phi(0)$. As a consequence Eqs. (A18) and (A19) still apply on condition to replace $\phi_{a}$ by $\phi_{0}$ where $\phi_{0}$ is given by solving the surface torque equation. In practice we are only interested in the asymptotic behaviour of the capacitance curve in the limit $1 / V \rightarrow 0$. In this regime, it can be shown that Eq. (C5) still holds and that the asymptotic behaviour of the capacitance curve is the same as in an antiparallel sample (see Eq. $(\mathrm{C} 7))$.

These results can be used to measure the ratio $l_{p} / d$. By denoting by $\bar{\phi}_{a}$ the value of $\phi_{a}$ measured by using the simplified Equation (A21), one obtains

$$
\frac{l_{p}}{d}=\frac{\phi_{a}-\bar{\phi}_{a}}{2 \gamma\left(I-\bar{\phi}_{a}\right)} \approx \frac{\phi_{a}-\bar{\phi}_{a}}{2 I \gamma}
$$

In our experiments with the $8 \mathrm{CB}$ we found that $\bar{\phi}_{a} \approx \phi_{a}$ to better than $0.1^{\circ}$. That means that $\gamma I l_{p} / d \leq 8.710^{-4}$. In our experiment, $d \approx 25 \mu \mathrm{m}$ which gives $l_{p}(n m) \leq 22 / \gamma$ knowing that $I \sim 1$. This is quite compatible with the values measured on rubbed polyimide by Faetti and Marianelli in a similar product, 5CB [47] (the values given by these authors range between $10 \mathrm{~nm}$ at room temperature and $50 \mathrm{~nm}$ close to the clearing temperature).

In the mixture $8 \mathrm{CB} / \mathrm{CB} 7 \mathrm{CB}$, we found $\phi_{a} \approx 1.8^{\circ}$ (see Fig. $20 \mathrm{~b}$ ) and $\bar{\phi}_{a} \approx 1.5^{\circ}$ (see Fig. A1) by using a $26-\mu \mathrm{m}$-thick sample. These values are independent of temperature within our experimental precision, which means that $I \gamma \frac{l_{p}}{d}$ is approximately constant, of the order of 0.0026 . This shows that $l_{p}$ varies approximatively as $1 / \gamma I$ as a function of temperature. This variation is shown in Fig. C1 and resembles very much the curve measured in 5CB by Faetti and Marianelli in 5CB [47]. These measurement show that the anchoring is weaker in the mixture $8 \mathrm{CB} / \mathrm{CB} 7 \mathrm{CB}$ than in pure $8 \mathrm{CB}$. We emphasize that with these values of $l_{p}$, it is possible to check that $\phi_{0}$ changes by less than $0.2^{\circ}$ in antiparrallel samples of thickness $d \approx 25 \mu \mathrm{m}$ when $V$ is varied from 0 to $V_{c}$. This justifies a posteriori our measurements of $\phi_{a}$ presented in \& 5.3 of the main text.

We end this discussion by noting that measuring $\bar{\phi}_{a}$ as a function of $1 / d$ should give a line whose the extrapolation to 0 gives $\phi_{a}$ and the slope $2 I \gamma l_{p}$. This could be a good method to measure both $\phi_{a}$ and $l_{p}$ on condition to know $\kappa$ and $\gamma$. 


\section{C.2 Role of finite surface anchoring in the optical measurements}

By using the optical method, we have assumed that the anchoring was infinitely strong. This assumption is clearly valid in the case of $8 \mathrm{CB}$, but we may question about its validity in the case of the mixture $8 \mathrm{CB} / \mathrm{CB} 7 \mathrm{CB}$. To test this point and estimate our error, we redid the calculations by taking into account the anchoring energy on the plate treated for planar anchoring. In that case, the bulk torque equation (2) must be solved with the new boundary condition

$$
l_{p}\left[\cos ^{2} \phi(0)+\kappa_{31} \sin ^{2} \phi(0)\right] \phi^{\prime}(0)=\frac{1}{2} \sin \left[2 \phi(0)-2 \phi_{a}\right]
$$

on the plate treated for planar anchoring. By taking the values of $l_{p}$ given in Fig. C1, we found that the values of $\kappa_{31}$ were a bit larger by 5 to $10 \%$ depending on temperature, which is negligible in view of our uncertainties.

Note than, in a similar way, the anchoring energy should also be taken into account on the plate treated for homeotropic anchoring. However, this effect should be substantially smaller because the elastic torque on this plate is proportional to $K_{3}$, which is very small with respect to $K_{1}$ in the mixture $8 \mathrm{CB} / \mathrm{CB} 7 \mathrm{CB}$. 